\title{
A robust and efficient radial return algorithm based on incremental energy minimization for the 3D Souza-Auricchio model for shape memory alloys
}

\author{
Giulia Scaleta ${ }^{\mathrm{a} b}$, Michaël Peigney ${ }^{\mathrm{c}}$ \\ ${ }^{a}$ Corresponding author. Department of Civil Engineering and Architecture, University of \\ Pavia, via Ferrata 3, 27100 Pavia, Italy \\ ${ }^{b}$ Laboratoire de Mécanique des Solides, Ecole Polytecnique, CNRS, Université \\ Paris-Saclay, 91128 Palaiseau, France \\ ${ }^{c}$ Laboratoire Navier, UMR 8205, Ecole des Ponts, IFSTTAR, CNRS, UPE, \\ Champs-sur-Marne, France
}

\begin{abstract}
The present paper focuses on the numerical simulation of quasi-static problems involving shape memory alloy (SMA) structures or components. Phenomenological constitutive models formulated within the continuum thermodynamics with internal variable framework describe phase transformation in a SMA by introducing a suitable set of internal variables, which may be constrained to satisfy a set of inequalities. The numerical treatment of such constraints, together with the presence of non-smooth functions and/or complementary conditions in the model formulation, is not an easy task and strongly influences the numerical convergence, algorithm robustness, and computational times. The aim of this paper is to propose a novel stateupdate procedure for the three-dimensional phenomenological model known as the Souza-Auricchio model. The proposed radial return algorithm, relying on an incremental energy minimization approach, allows for an easy implementation of model equations and internal constraints and avoids the use of regularization parameters for the treatment of non-smooth functions. Several numerical simulations assess the noticeable efficiency, robustness, and performance of the proposed approach, while comparisons with a classical algorithm proposed in the literature show the reduced computational times.
\end{abstract}

Keywords: shape memory alloys, numerical scheme, constitutive modeling, incremental energy minimization, radial return 


\section{Introduction}

Shape memory alloys (SMAs) are smart materials with the ability to recover large strains after thermal cycling or mechanical loading/unloading (Lagoudas, 2008). Thanks to these unique properties, denoted as shape memory effect and pseudoelasticity, SMA materials are employed in numerous commercial applications, ranging from the biomedical, aerospace, automotive, and earthquake engineering fields to recent technologies as additive manufacturing and active textiles (Jani et al., 2014).

Motivated by the ever-increasing use of SMA-based components and devices, a lot of research has been dedicated to the modeling, simulation, and experimental testing of these alloys.

Several constitutive models have been proposed in the literature for describing the complex behavior of SMAs; generally, they are categorized in macroscopic, microscopic, and micro-macro models. A description of all the modeling approaches is out of the present scope; the reader is referred to Cisse et al. (2016) for a recent review on the topic. Among the others, phenomenological macroscopic models formulated within the framework of continuum thermodynamics with internal variables attract large engineering interest. Starting from pioneering models involving only scalar internal variables (Tanaka and Nagaki, 1982), such models have actually reached high levels of accuracy and refinement (Auricchio et al., 2014a; Lagoudas et al., 2012; Zaki and Moumni, 2007). Most of the models are in fact able to describe several mechanisms and effects characterizing SMA behavior, e.g., martensite reorientation (Arghavani et al., 2010; Pan et al., 2007; Popov and Lagoudas, 2007), twinned-detwinned martensites (Auricchio and Bonetti, 2013), thermomechanical coupling (Auricchio et al., 2016), R-phase and anisotropy (Sedlák et al., 2012), asymmetric behavior in tension and compression (Auricchio et al., 2009b), transformation-induced plasticity (Hartl and Lagoudas, 2009; Auricchio et al., 2007), viscoplasticity (Chemisky et al., 2014), internal damage (Hartl et al., 2014), functional fatigue effects (Barrera et al., 2014), microstructure-dependent inelasticity (Grandi and Stefanelli, 2014), and the two-way shape memory effect (Lexcellent et al., 2000).

One of the major concerns associated to constitutive modeling is the need of suitable numerical algorithms to treat non-smooth functions and/or local constraints deriving from the set of nonlinear constitutive equations describing material behavior. The presence of equality/inequality constraints in the evolution problem and/or on internal variables as well as the introduc- 
tion of non-smooth functions in the model definition, often treated through regularized terms, may prevent numerical convergence and may influence numerical robustness, accuracy, and computational times. Therefore, a robust and accurate numerical implementation is of extreme importance for the design of novel structures (Peraza-Hernandez and Galvan, 2013) and for the development of novel technologies (Meisel et al., 2015). The implementation generally consists first in the time-integration of the system of constitutive equations and then in a state-update procedure to derive the updated variables.

Several models are available in a suitable form to perform simulations of complex SMA-based geometries subjected to general thermomechanical loading paths in both finite element (Arghavani et al., 2010; Sedlák et al., 2012; Zaki, 2012) and isogeometric analysis (Auricchio et al., 2015; Dhote et al., 2015) frameworks. The state-update procedures generally adopted to treat SMA constitutive equations are based on return-map schemes, incremental energy minimization approaches, or algorithms for mathematical programming.

A wide class of SMA models implements a return-map-like procedure in an implicit framework (Auricchio and Petrini, 2004; Qidwai and Lagoudas, 2000; Lagoudas et al., 2012; Zaki, 2012; Hartl and Lagoudas, 2009), even if some works have recently proposed implementations in an explicit environment (Stebner and Brinson, 2013; Scalet et al., 2015). The work by Popov and Lagoudas (2007) applies an extension of the closest-point projection algorithm to treat a SMA model incorporating single- and multi-variant martensites. The advantage in using such a procedure is granted by its good numerical performance and well-established numerical properties.

To eliminate the need for a predictor-corrector-type scheme and to omit an active set search, which may become elaborate in the presence of coupled evolution equations and internal constraints, Kiefer et al. (2012) presented two alternative algorithms for the integration of the constitutive equations for magnetic SMAs, namely, the classical return-map scheme and a FischerBurmeister-based algorithm. The work demonstrates a greater numerical efficiency of the Fischer-Burmeister-based algorithm, compared to the classical return-mapping. In the context of SMA micromechanical modeling, it is noteworthy to cite the contributions by Bartel and Hackl (2009, 2010); Bartel et al. (2011), who also employed the Fischer-Burmeister complementary function. Recently, Auricchio et al. (2014a) have proposed the use of the Fischer-Burmeister function to treat the inequality constraints on the internal 
variables and the Kuhn-Tucker complementary inequality conditions deriving from a plasticity-like model formulation. A regularized Fischer-Burmeister function is adopted to treat the non-differentiability of the function when both the arguments are equal to zero.

An alternative approach to return-map procedures is the class of variational methods, which relies on an incremental energy minimization approach. Sedlák et al. (2012) applied the Nelder-Mead minimization algorithm to solve the derived problem for SMAs and introduced a regularization energy to assure the fulfillment of constraints on internal variables. Stupkiewicz and Petryk (2013) presented a pseudoelastic model within the incremental energy minimization framework and proposed an unified augmented Lagrangian treatment of both constitutive constraints and non-smooth dissipation function.

Another approach consists in rewriting the problem as a mathematical programming problem which is solved using general optimization methods. Peigney et al. (2011) applied an incremental variational approach to SMAs and reformulated the incremental problem as a linear complementarity problem. The advantage of the formulation is to solve simultaneously the equilibrium equations and the constitutive laws, taking the internal constraints into account. The obtained formulation leads to a simple and efficient numerical algorithm, solved using interior-point methods.

In the present paper, we focus on the three-dimensional phenomenological model introduced by Souza et al. (1998), and then treated and generalized in Auricchio and Petrini (2004); Evangelista et al. (2009) (hence the denomination Souza-Auricchio model, in the following). The model has received a large attention thanks to several advantageous features (Grandi and Stefanelli, 2015), such as a simple plasticity-like formulation, few parameters, and a robust numerical implementation, which have allowed its application to the simulation of a wide range of devices (Auricchio et al., 2014b). Several works have focused on the numerical implementation of this model, to treat the evolution of the tensorial internal variable (i.e., the transformation strain), subjected to a saturation constraint. Souza et al. (1998) adopted a return-map scheme and used a criterion for the nucleation of the product phase, while Auricchio and Petrini (2004) used a regularized parameter to treat the case of vanishing transformation strain and Jähne (2012) proposed an explicit integration scheme. Recently, Artioli and Bisegna (2015) have adopted an incremental energy minimization approach for the solution of the constitutive equations, without introducing any regularization, and 
have shown the effect of the regularized term on material response. The use of a regularization parameter strongly influences the predicted response at the cost of accuracy. Arghavani et al. (2011b) proposed an integration algorithm for a finite-strain extension of the Souza-Auricchio model, based on a logarithmic mapping and a nucleation-completion criterion, while Arghavani et al. (2011a) proposed an improved alternative constitutive model for a finite-strain extension of the Souza-Auricchio model, expressed in terms of only symmetric tensors.

The present paper aims to propose a novel solution algorithm for the Souza-Auricchio model. Among the several numerical approaches cited above, the proposed algorithm belongs to the class of variational methods relying on an incremental energy minimization approach. The evolution of the transformation strain in a finite time step incrementally minimizes a convex functional, given by the sum of the elastic energy and the dissipation function. The treatment is then based on a radial-return algorithm and a standard Newton-Raphson scheme is adopted to solve the single scalar equation in both the unsaturated and saturated phase transformation cases. The solution algorithm as well as the initial guess for the resultant nonlinear equation are also discussed. The formulation is here restricted to the framework of infinitesimal strain theory; however, the present approach can be applied to geometric nonlinear problems.

The choice of using this approach is due to its suitable variational structure which facilitates the treatment of internal constraints and allows for an efficient numerical implementation. The advantages of the proposed algorithm are its equation simplicity, easy implementation, the possibility of avoiding regularized terms in both energy/dissipation definition and transformation strain norm, and, overall, the reduced computational times. The actual nonlinear system consists of only one scalar equation for both the unsaturated and phase transformation cases. Further simplifications are possible for low-temperature cases, i.e. when temperature is lower than the equilibrium temperature $T^{*}$. This results in noticeable low computational times and costs, which are of great important in engineering simulations.

To test the performance, robustness, and efficiency of the proposed algorithm several finite element analyses are presented. The simulations range from classical uniaxial and biaxial tests to more complex multiaxial problems, reproducing the pseudoelastic and shape memory properties. Moreover, the algorithm in Auricchio and Petrini (2004) is implemented to provide a comparison in terms of model response, computational times, as well as iteration 
number and to highlight the advantages in using the proposed radial return algorithm.

The paper is organized as follows. Section 2 briefly reviews the continuum formulation of the Souza-Auricchio model, together with the related boundary value problem. Section 3 presents the equations in the time-discrete framework and Section 4 describes the proposed algorithmic scheme. Then, Section 5 presents the results of several numerical simulations. Finally, conclusions are given in Section 6 .

\section{Continuum model formulation}

\subsection{Constitutive laws}

We briefly reviews the time-continuous formulation of the Souza-Auricchio model. The reader is referred to Souza et al. (1998) for details.

The assumed control variables are the total strain $\varepsilon$ and the absolute temperature $T$, while the internal variable is the transformation strain $\boldsymbol{e}^{t r}$. According to experimental evidences (Wayman, 1989), $\boldsymbol{e}^{t r}$ is assumed to be traceless and satisfies the saturation constraint:

$$
\left\|e^{t r}\right\| \leq \varepsilon_{L}
$$

where $\varepsilon_{L}$ is a material parameter corresponding to the scalar maximum transformation strain reached at the end of the phase transformation during an uniaxial test and $\|\cdot\|$ denotes the Euclidean norm.

The Helmoltz free energy function $\Psi=\Psi\left(\varepsilon, T, \boldsymbol{e}^{t r}\right)$ is expressed as follows:

$$
\Psi=\frac{1}{2} \kappa \theta^{2}+G\left\|\boldsymbol{e}-\boldsymbol{e}^{t r}\right\|^{2}+\tau_{M}\left\|\boldsymbol{e}^{t r}\right\|+\frac{1}{2} h\left\|\boldsymbol{e}^{t r}\right\|^{2}+\mathcal{I}_{\varepsilon_{L}}\left(\boldsymbol{e}^{t r}\right) .
$$

Here, $\theta$ and $\boldsymbol{e}$ are the volumetric and deviatoric part of $\boldsymbol{\varepsilon}$, respectively; different expressions can be chosen for $\tau_{M}$ : in this case we assume $\tau_{M}=\beta\left\langle T-T^{*}\right\rangle$, where $\beta$ is a positive parameter related to the dependence of the critical stress on temperature, $T^{*}$ is a reference temperature, and $\langle\cdot\rangle$ indicates the positive part function; $\kappa$ and $G$ are the bulk and shear modulus, respectively; $h$ defines the phase transformation hardening. The indicator function

$$
\mathcal{I}_{\varepsilon_{L}}\left(\boldsymbol{e}^{t r}\right)=\left\{\begin{array}{cl}
0 & \text { if }\left\|\boldsymbol{e}^{t r}\right\| \leq \varepsilon_{L} \\
+\infty & \text { otherwise }
\end{array}\right.
$$

is introduced to satisfy the transformation strain constraint (1). 
Following standard arguments (Gurtin, 1981; Coleman and Noll, 1963), the constitutive equations are:

$$
\begin{aligned}
& p=\frac{\partial \Psi}{\partial \theta}=\kappa \theta \\
& \boldsymbol{s}=\frac{\partial \Psi}{\partial \boldsymbol{e}}=2 G\left(\boldsymbol{e}-\boldsymbol{e}^{t r}\right), \\
& \boldsymbol{X}=-\frac{\partial \Psi}{\partial \boldsymbol{e}^{t r}}=\boldsymbol{s}-\tau_{M} \partial\left\|\boldsymbol{e}^{t r}\right\|-h \boldsymbol{e}^{t r}-\gamma \frac{\boldsymbol{e}^{t r}}{\left\|\boldsymbol{e}^{t r}\right\|},
\end{aligned}
$$

where $p$ and $\boldsymbol{s}$ are the volumetric and deviatoric part of $\boldsymbol{\sigma}$, respectively, and $\boldsymbol{X}$ is the thermodynamic stress-like quantity associated to $\boldsymbol{e}^{t r}$. If $\boldsymbol{e}^{t r} \neq \mathbf{0}$, the subdifferential $\partial\left\|\boldsymbol{e}^{t r}\right\|$ in (4) is given by

$$
\partial\left\|e^{t r}\right\|=\frac{e^{t r}}{\left\|e^{t r}\right\|} .
$$

If $\boldsymbol{e}^{t r}=\mathbf{0}$, we have

$$
\partial\left\|\boldsymbol{e}^{t r}\right\|=\{\tau \mid \operatorname{tr} \tau=0 ;\|\tau\| \leq 1\} .
$$

Note that $\partial\left\|\boldsymbol{e}^{t r}\right\|$ is not single-valued when $\boldsymbol{e}^{t r}=\mathbf{0}$. The variable $\gamma$ in (4) results from the indicator function subdifferential $\partial \mathcal{I}_{\varepsilon_{L}}\left(\boldsymbol{e}^{t r}\right)$ and it is defined as follows:

$$
\gamma=\left\{\begin{array}{cl}
0 & \text { if }\left\|\boldsymbol{e}^{t r}\right\|<\varepsilon_{L} \\
\geq 0 & \text { if }\left\|\boldsymbol{e}^{t r}\right\|=\varepsilon_{L}
\end{array},\right.
$$

with $\partial \mathcal{I}_{\varepsilon_{L}}\left(\boldsymbol{e}^{t r}\right)=\gamma \boldsymbol{e}^{t r} /\left\|\boldsymbol{e}^{t r}\right\|$.

To describe phase transformation and inelasticity evolution, a Mises-type limit function $F=F(\boldsymbol{X})$ is introduced in the following form:

$$
F=\|\boldsymbol{X}\|-R_{Y},
$$

where $R_{Y}$ is a positive material parameter corresponding to the radius of the elastic domain in the deviatoric stress space.

The evolution equation for $\boldsymbol{e}^{t r}$ takes the form:

$$
\dot{\boldsymbol{e}}^{t r}=\dot{\lambda} \frac{\partial F}{\partial \boldsymbol{X}}=\dot{\lambda} \frac{\boldsymbol{X}}{\|\boldsymbol{X}\|},
$$

where $\dot{\lambda}$ is the non-negative consistency parameter. The model is then completed by the classical Kuhn-Tucker conditions:

$$
\dot{\lambda} \geq 0, \quad F \leq 0, \quad \dot{\lambda} F=0 .
$$




\subsection{Boundary value problem}

Consider a three-dimensional continuum occupying a domain $\Omega$ and subjected to a prescribed loading history. The continuum is assumed to be at mechanical and thermal equilibrium at each time. In such a situation, the temperature field is homogeneous at each time and takes a prescribed value $T(t)$. The stress field $\boldsymbol{\sigma}$ satisfies the equilibrium equation

$$
\begin{gathered}
\operatorname{div} \boldsymbol{\sigma}+\boldsymbol{f}^{d}(t)=\mathbf{0} \text { in } \Omega, \\
\boldsymbol{\sigma} \cdot \boldsymbol{n}=\boldsymbol{T}^{d}(t) \text { on } \partial \Omega_{T},
\end{gathered}
$$

where $\boldsymbol{f}^{d}(t)$ are body forces in $\Omega$ and $\boldsymbol{T}^{d}(t)$ are tractions specified on a part $\partial \Omega_{T}$ of the boundary $\partial \Omega$. The strain field $\boldsymbol{\varepsilon}$ derives from a kinematically admissible displacement field $\boldsymbol{u}$, i.e.,

$$
\begin{gathered}
\boldsymbol{\varepsilon}=\frac{1}{2}\left(\nabla \boldsymbol{u}+\nabla^{T} \boldsymbol{u}\right) \text { in } \Omega \\
\boldsymbol{u}=\boldsymbol{u}^{d}(t) \text { on } \partial \Omega_{u},
\end{gathered}
$$

where $\boldsymbol{u}^{d}(t)$ are given displacements on $\partial \Omega_{u}=\partial \Omega-\partial \Omega_{T}$. The functions $\boldsymbol{f}^{d}, \boldsymbol{T}^{d}$, and $\boldsymbol{u}^{d}$ may also depend on the position $\boldsymbol{x}$, but that dependence is omitted in the notations.

Together, the local constitutive relations (4-8) and the field equations (910) define a boundary value problem that governs the evolution of the SMA continuum. In general, a discretization both in space and time is necessary to solve that boundary value problem, as discussed next.

\section{Discrete model formulation}

The time-discretization consists in introducing a finite number of time instants $t_{0}<\cdots<t_{N}$ and estimating the solution at each time $t_{i}$ using a timemarching approach. To do so, the crucial step is to introduce an incremental problem for estimating the values $\left(\boldsymbol{u}_{n+1}, \boldsymbol{e}_{n+1}^{t r}\right)$ at $t=t_{n+1}$, assuming the values $\left(\boldsymbol{u}_{n}, \boldsymbol{e}_{n}^{t r}\right)$ at $t=t_{n}$ known. A widely used time-discretization scheme is the Euler implicit scheme, which in the present context corresponds the incremental problem defined as follows:

$$
\begin{gathered}
\boldsymbol{u}_{n+1} \in \mathcal{K}_{u}\left(t_{n+1}\right), \quad \boldsymbol{\sigma}_{n+1} \in \mathcal{K}_{\sigma}\left(t_{n+1}\right), \quad \boldsymbol{e}_{n+1}^{t r} \in \mathcal{K}_{\varepsilon_{L}} \\
p_{n+1}=\kappa \theta_{n+1}, \boldsymbol{s}_{n+1}=2 G\left(\boldsymbol{e}_{n+1}-\boldsymbol{e}_{n+1}^{t r}\right)
\end{gathered}
$$




$$
\begin{gathered}
\boldsymbol{X}_{n+1}=\boldsymbol{s}_{n+1}-\tau_{M, n+1} \partial\left\|\boldsymbol{e}_{n+1}^{t r}\right\|-h \boldsymbol{e}_{n+1}^{t r}-\gamma_{n+1} \frac{\boldsymbol{e}_{n+1}^{t r}}{\left\|\boldsymbol{e}_{n+1}^{t r}\right\|}, \\
\boldsymbol{e}_{n+1}^{t r}=\boldsymbol{e}_{n}^{t r}+\Delta \lambda \frac{\boldsymbol{X}_{n+1}}{\left\|\boldsymbol{X}_{n+1}\right\|}, \\
\gamma_{n+1} \geq 0,\left\|\boldsymbol{e}_{n+1}^{t r}\right\| \leq \varepsilon_{L}, \gamma_{n+1}\left(\left\|\boldsymbol{e}_{n+1}^{t r}\right\|-\varepsilon_{L}\right)=0 \\
\Delta \lambda \geq 0,\left\|\boldsymbol{X}_{n+1}\right\| \leq R_{Y}, \Delta \lambda\left(R_{Y}-\left\|\boldsymbol{X}_{n+1}\right\|\right)=0 .
\end{gathered}
$$

In (11.1), the sets of admissible fields are defined by

$$
\begin{aligned}
& \mathcal{K}_{\varepsilon_{L}}=\left\{\boldsymbol{e}^{t r} \mid\left\|\boldsymbol{e}^{t r}\right\| \leq \varepsilon_{L} \text { for all } \boldsymbol{x} \in \Omega\right\}, \\
& \mathcal{K}_{u}(t)=\left\{\boldsymbol{u} \mid \boldsymbol{u}=\boldsymbol{u}^{d}(t) \text { on } \partial \Omega_{u}\right\} \\
& \mathcal{K}_{\sigma}(t)=\left\{\boldsymbol{\sigma} \mid \operatorname{div} \boldsymbol{\sigma}+\boldsymbol{f}^{d}(t)=0 \text { in } \Omega ; \boldsymbol{\sigma} . \boldsymbol{n}=\boldsymbol{T}^{d}(t) \text { on } \partial \Omega_{T}\right\} .
\end{aligned}
$$

The incremental problem (11) being nonlinear, the issues of existence and uniqueness of solutions need to be addressed. In that regard, it is useful to observe that a variational formulation is attached to the incremental problem (11). Consider indeed the functional $\mathcal{F}\left(\boldsymbol{u}, \boldsymbol{e}^{t r}\right)$ defined as the sum $\mathcal{F}\left(\boldsymbol{u}, \boldsymbol{e}^{t r}\right)=$ $\mathcal{F}^{e}\left(\boldsymbol{u}, \boldsymbol{e}^{t r}\right)+\mathcal{F}^{d}\left(\boldsymbol{e}^{t r}\right)$ of the elastic potential energy

$$
\mathcal{F}^{e}\left(\boldsymbol{u}, \boldsymbol{e}^{t r}\right)=\int_{\Omega} \Psi\left(\varepsilon, T\left(t_{n+1}\right), \boldsymbol{e}^{t r}\right) d \omega-\int_{\Omega} \boldsymbol{f}^{d} \cdot \boldsymbol{u} d \omega-\int_{\partial \Omega_{T}} \boldsymbol{T}^{d} \cdot \boldsymbol{u} d a
$$

and the dissipative term

$$
\mathcal{F}^{d}\left(\boldsymbol{e}^{t r}\right)=R_{Y} \int_{\Omega}\left\|\boldsymbol{e}^{t r}-\boldsymbol{e}_{n}^{t r}\right\| d \omega
$$

Using standard results in convex analysis (Brézis, 1972; Rockafellar, 1970), it can be proved (see Appendix A) that the solutions to (11) are minimizers of $\mathcal{F}$, i.e. the incremental problem (11) is formally equivalent to the minimization problem

$$
\inf _{\left(\boldsymbol{u}, \boldsymbol{e}^{t r}\right) \in \mathcal{K}_{u}\left(t_{n+1}\right) \times \mathcal{K}_{\varepsilon_{L}}} \mathcal{F}\left(\boldsymbol{u}, \boldsymbol{e}^{t r}\right)
$$

Variational formulations governing the evolution of generalized standard materials have been largely used in the literature in case of convex potentials and have been also extended to non-convex potentials to predict microstructure formation (Ortiz and Repetto, 1999; Miehe et al., 2002; Carstensen et al., 
2002). In the present case observe that the function $\mathcal{F}$ is convex in $\left(\boldsymbol{u}, \boldsymbol{e}^{t r}\right)$, with quadratic growth at infinity. It follows that minimizers of $\mathcal{F}$ do exist, provided adequate functional spaces are chosen for $\mathcal{K}_{u}$ and $\mathcal{K}_{\varepsilon_{L}}$. For a three-dimensional continuum, such a choice of appropriate functional spaces is a subtle mathematical issue that is beyond the scope of this paper. The situation simplifies once space discretization is performed: in practice, the spaces $\mathcal{K}_{u}$ and $\mathcal{K}_{\varepsilon_{L}}$ are indeed taken as finite-dimensional, using, for instance, the finite element method. In such case, the aforementioned properties of $\mathcal{F}$ ensures that the (space-discretized) incremental problem does admit some solutions. If $h>0$ (which is assumed in the following) then $\mathcal{F}$ is actually strictly convex, which ensures that the minimizer is unique.

Several strategies are available for solving the minimization problem (15). A possible approach consists in rewriting the minimization problem (15) in the nested form

$$
\inf _{\boldsymbol{u} \in \mathcal{K}_{u}\left(t_{n+1}\right)}\left\{\inf _{\boldsymbol{t a n}^{t r} \in \mathcal{K}_{\varepsilon_{L}}} \mathcal{F}\left(\boldsymbol{u}, \boldsymbol{e}^{t r}\right)\right\}
$$

Observing that the variable $\boldsymbol{e}^{t r}$ in (16) is free from any differential constraint, we have

$$
\inf _{e^{t r} \in \mathcal{K}_{\varepsilon_{L}}} \mathcal{F}\left(\boldsymbol{u}, e^{t r}\right)=\int_{\Omega} \Psi_{n+1}(\varepsilon) d \omega-\int_{\Omega} \boldsymbol{f}^{d} \cdot \boldsymbol{u} d \omega-\int_{\partial \Omega_{T}} \boldsymbol{T}^{d} \cdot \boldsymbol{u} d a .
$$

where the function

$$
\Psi_{n+1}(\varepsilon)=\inf _{e^{t r}:\left\|e^{t r}\right\| \leq \varepsilon_{L}} \Psi\left(\varepsilon, T\left(t_{n+1}\right), e^{t r}\right)+R_{Y}\left\|e^{t r}-e_{n}^{t r}\right\|
$$

is obtained by solving a local nonlinear problem. Using (17), Eq. (16) can be rewritten as

$$
\inf _{\boldsymbol{u} \in \mathcal{K}_{u}\left(t_{n+1}\right)} \int_{\Omega} \Psi_{n+1}(\varepsilon) d \omega-\int_{\Omega} \boldsymbol{f}^{d} \cdot \boldsymbol{u} d \omega-\int_{\partial \Omega_{T}} \boldsymbol{T}^{d} \cdot \boldsymbol{u} d a
$$

The functional that appears in (19) is similar to the functional $\mathcal{F}^{e}$ in (13), the only difference being that the Helmoltz free energy $\Psi$ in (2) is replaced by the function $\Psi_{n+1}$. Solving (19) amounts to solve the equilibirum problem of an elastic nonlinear continuum with a free energy density $\Psi_{n+1}$, i.e., obeying the constitutive law $\boldsymbol{\sigma}=\Psi_{n+1}^{\prime}(\varepsilon)$. Note that $\Psi_{n+1}$ is path-dependent since it depends on the state $\boldsymbol{e}_{n}^{t r}$ at the previous time instant.

From a numerical point of view, Eq. (19) is a global nonlinear minimization problem that can be solved using a descend algorithm or a Newton 
method. Such algorithms make use of the first and second derivatives of the function to minimize, which in the present case amounts to calculate $\Psi_{n+1}^{\prime}(\varepsilon)$ and the consistent tangent operator $\Psi_{n+1}^{\prime \prime}(\varepsilon)$. In the following we propose an efficient algorithm for calculating those quantities.

\section{A radial return algorithm}

In the present section, we focus on the local nonlinear problem (18) which, as explained before, is the central building block for solving the incremental structural problem (11). To alleviate the expressions, the term $\tau_{M, n+1}$ is denoted by $\tau_{M}$ in the following. Replacing $\Psi$ with its expression (2), the minimization problem in (18) can be rewritten as

$$
\inf _{\boldsymbol{e}^{t r}} G^{\prime}\left\|\boldsymbol{e}^{t r}\right\|^{2}-2 G^{\prime} \boldsymbol{e}^{t r}: \boldsymbol{e}^{\prime}+\tau_{M}\left\|\boldsymbol{e}^{t r}\right\|+R_{Y}\left\|\boldsymbol{e}^{t r}-\boldsymbol{e}_{n}^{t r}\right\|+\mathcal{I}_{\varepsilon_{L}}\left(\boldsymbol{e}^{t r}\right)
$$

where $G^{\prime}=G+h / 2$ and $\boldsymbol{e}^{\prime}=\left(G / G^{\prime}\right) \boldsymbol{e}$. The function to minimize in (20) is strictly convex in $\boldsymbol{e}^{t r}$ and therefore admits a unique solution. That solution, denoted by $\boldsymbol{e}_{n+1}^{t r}$ in the following, is characterized by the optimality equation

$$
\mathbf{0} \in 2 G^{\prime}\left(\boldsymbol{e}_{n+1}^{t r}-\boldsymbol{e}^{\prime}\right)+\tau_{M} \partial\left\|\boldsymbol{e}_{n+1}^{t r}\right\|+R_{Y} \partial\left\|\boldsymbol{e}_{n+1}^{t r}-\boldsymbol{e}_{n}^{t r}\right\|+\gamma_{n+1} \frac{\boldsymbol{e}_{n+1}^{t r}}{\left\|\boldsymbol{e}_{n+1}^{t r}\right\|}
$$

where

$$
\begin{array}{r}
\gamma_{n+1} \geq 0,\left\|\boldsymbol{e}_{n+1}^{t r}\right\| \leq \varepsilon_{L}, \gamma_{n+1}\left(\left\|\boldsymbol{e}_{n+1}^{t r}\right\|-\varepsilon_{L}\right)=0, \\
\partial\left\|\boldsymbol{e}_{n+1}^{t r}-\boldsymbol{e}_{n}^{t r}\right\|= \begin{cases}\frac{\boldsymbol{e}_{n+1}^{t r}-\boldsymbol{e}_{n}^{t r}}{\left\|\boldsymbol{e}_{n+1}^{t r}-\boldsymbol{e}_{n}^{t r}\right\|} & \text { if } \boldsymbol{e}_{n+1}^{t r} \neq \boldsymbol{e}_{n}^{t r} \\
\{\tau: \operatorname{tr} \tau=0,\|\tau\| \leq 1\} & \text { if } \boldsymbol{e}_{n+1}^{t r}=\boldsymbol{e}_{n}^{t r},\end{cases}
\end{array}
$$

and, similarly,

$$
\partial\left\|\boldsymbol{e}_{n+1}^{t r}\right\|= \begin{cases}\frac{\boldsymbol{e}_{n+1}^{t r}}{\left\|\boldsymbol{e}_{n+1}^{t r}\right\|} & \text { if } \boldsymbol{e}_{n+1}^{t r} \neq \mathbf{0} \\ \{\tau: \operatorname{tr} \tau=0,\|\tau\| \leq 1\} & \text { if } \boldsymbol{e}_{n+1}^{t r}=\mathbf{0}\end{cases}
$$

From a numerical standpoint, there are two possible approaches for solving (20). The first approach consists in constructing a minimizing sequence, using for instance a linear search algorithm. Since we are in the most favorable situation where the function to minimize is convex, convergence of such algorithms is ensured under mild conditions. The second approach consists in solving the nonlinear equation (21), using for instance a Newton-Raphson 
procedure. Generally speaking, the convergence of the Newton-Raphson procedure is conditioned by the choice of the initial guess. In the case where the initial guess is close enough to the solution, the Newton-Raphson procedure converges very fast - usually faster than linear search algorithms or the like - and it is hard to beat. In the following, we favor the second approach, i.e., solving the optimality equation (21). The reason is that Eq. (21) can be reduced to a scalar equation, as detailed next. For scalar problems, the issue of finding a good initial guess simplifies considerably, which allows one to take full advantage of the Newton-Raphson procedure.

\subsection{Check for elastic evolution or fully reversed phase transformation}

To solve (21), the first step consists in checking whether $\boldsymbol{e}_{n+1}^{t r}=\boldsymbol{e}_{n}^{t r}$ or $\boldsymbol{e}_{n+1}^{t r}=\mathbf{0}$. This is accomplished using the expressions (23-24). The obtained conditions take different expressions whether $\boldsymbol{e}_{n}^{t r} \neq \mathbf{0}$ or $\boldsymbol{e}_{n}^{t r}=\mathbf{0}$ :

- Case $\boldsymbol{e}_{n}^{t r} \neq \mathbf{0}$ :

We have $\boldsymbol{e}_{n+1}^{t r}=\boldsymbol{e}_{n}^{t r}$ (elastic evolution) if and only if

$$
\left\|\boldsymbol{a}-\tau_{M} \frac{\boldsymbol{e}_{n}^{t r}}{\left\|\boldsymbol{e}_{n}^{t r}\right\|}\right\| \leq R_{Y}
$$

We have $\boldsymbol{e}_{n+1}^{t r}=\mathbf{0}$ (fully reversed phase transformation) if and only if

$$
\left\|2 G^{\prime} \boldsymbol{e}^{\prime}+R_{Y} \frac{\boldsymbol{e}_{n}^{t r}}{\left\|\boldsymbol{e}_{n}^{t r}\right\|}\right\| \leq \tau_{M}
$$

- Case $\boldsymbol{e}_{n}^{t r}=\mathbf{0}$ :

We have $\boldsymbol{e}_{n+1}^{t r}=\mathbf{0}$ if and only if

$$
\|\boldsymbol{a}\| \leq R_{Y}+\tau_{M}
$$

In (26-27), $\boldsymbol{a}$ is the trial elastic state, defined by

$$
\boldsymbol{a}=2 G^{\prime}\left(\boldsymbol{e}^{\prime}-\boldsymbol{e}_{n}^{t r}\right)
$$

\subsection{Unsaturated phase transformation}

If none of the inequalities (25-27) is satisfied, then $\boldsymbol{e}_{n+1}^{t r} \notin\left\{\mathbf{0}, \boldsymbol{e}_{n}^{t r}\right\}$ and Eq. (21) becomes

$$
\mathbf{0}=2 G^{\prime}\left(\boldsymbol{e}_{n+1}^{t r}-\boldsymbol{e}^{\prime}\right)+\tau_{M} \frac{\boldsymbol{e}_{n+1}^{t r}}{x}+R_{Y} \frac{\boldsymbol{e}_{n+1}^{t r}-\boldsymbol{e}_{n}^{t r}}{\left\|\boldsymbol{e}_{n+1}^{t r}-\boldsymbol{e}_{n}^{t r}\right\|}+\gamma_{n+1} \frac{\boldsymbol{e}_{n+1}^{t r}}{\left\|\boldsymbol{e}_{n+1}^{t r}\right\|}
$$


with $x=\left\|\boldsymbol{e}_{n+1}^{t r}\right\|$ and $\gamma_{n+1}$ satisfying (22). To solve (29), first consider the equation

$$
\mathbf{0}=2 G^{\prime}\left(\boldsymbol{e}_{n+1}^{t r}-\boldsymbol{e}^{\prime}\right)+\tau_{M} \frac{\boldsymbol{e}_{n+1}^{t r}}{x}+R_{Y} \frac{\boldsymbol{e}_{n+1}^{t r}-\boldsymbol{e}_{n}^{t r}}{\left\|\boldsymbol{e}_{n+1}^{t r}-\boldsymbol{e}_{n}^{t r}\right\|}
$$

which amounts to drop the constraint $\left\|\boldsymbol{e}^{t r}\right\| \leq \varepsilon_{L}$. Eq. (30) can written as

$$
\mathbf{0}=2 G^{\prime}\left(\boldsymbol{e}_{n+1}^{t r}-\boldsymbol{e}_{n}^{t r}\right)-\boldsymbol{a}+\tau_{M} \frac{\boldsymbol{e}_{n+1}^{t r}-\boldsymbol{e}_{n}^{t r}}{x}+\tau_{M} \frac{\boldsymbol{e}_{n}^{t r}}{x}+R_{Y} \frac{\boldsymbol{e}_{n+1}^{t r}-\boldsymbol{e}_{n}^{t r}}{\left\|\boldsymbol{e}_{n+1}^{t r}-\boldsymbol{e}_{n}^{t r}\right\|} .
$$

Hence

$$
\left(2 G^{\prime}+\frac{\tau_{M}}{x}+\frac{R_{Y}}{\left\|\boldsymbol{e}_{n+1}^{t r}-\boldsymbol{e}_{n}^{t r}\right\|}\right)\left(\boldsymbol{e}_{n+1}^{t r}-\boldsymbol{e}_{n}^{t r}\right)=\boldsymbol{b}
$$

where

$$
\boldsymbol{b}=\boldsymbol{a}-\frac{\tau_{M}}{x} \boldsymbol{e}_{n}^{t r}
$$

Eq. (31) shows that the tensor $\boldsymbol{e}_{n+1}^{t r}-\boldsymbol{e}_{n}^{t r}$ is parallel to $\boldsymbol{b}$. Taking the norm of (31) and using the fact that $G^{\prime}, \tau_{M}, x, R_{Y}$ are positive, we find

$$
\left\|\boldsymbol{e}_{n+1}^{t r}-\boldsymbol{e}_{n}^{t r}\right\|=\frac{\|\boldsymbol{b}\|-R_{Y}}{\tau_{M} / x+2 G^{\prime}}
$$

Substituting in (31), we finally arrive at

$$
\boldsymbol{e}_{n+1}^{t r}=\boldsymbol{e}_{n}^{t r}+\frac{\boldsymbol{b}}{\|\boldsymbol{b}\|}\left(\frac{\|\boldsymbol{b}\|-R_{Y}}{\tau_{M} / x+2 G^{\prime}}\right) .
$$

Eq. (33) shows that $\boldsymbol{e}_{n+1}^{t r}$ is entirely determined by the positive scalar $x$. We are thus left with the issue of finding $x$. This is accomplished by taking the norm of (33), leading to the consistency equation

$$
x=\left\|\boldsymbol{e}_{n}^{t r}+\frac{\boldsymbol{b}}{\|\boldsymbol{b}\|}\left(\frac{\|\boldsymbol{b}\|-R_{Y}}{\tau_{M} / x+2 G^{\prime}}\right)\right\|
$$

in which $x$ is the only unknown. Eq. (34) can be rewritten in the form

$$
f(x)=0
$$

where

$$
f(x)=\|\boldsymbol{c}\|-\tau_{M}-2 G^{\prime} x
$$


and

$$
\boldsymbol{c}=2 G^{\prime} \boldsymbol{e}^{\prime}-R_{Y} \frac{\boldsymbol{b}}{\|\boldsymbol{b}\|}
$$

The equation $f(x)=0$ has a unique solution in $] 0,+\infty[$. More precisely, we have

$$
\lim _{x \rightarrow 0^{+}} f(x)>0 ; f\left(x_{\max }\right) \leq 0
$$

where $x_{\max }=\left\|\boldsymbol{e}^{\prime}\right\|+\left(R_{Y}-\tau_{M}\right) / 2 G^{\prime}$. The first inequality in (37) follows from the assumption that none of the inequalities (25-27) are satisfied. To obtain the second inequality in (37), observe from (36) that $\|\boldsymbol{c}\| \leq 2 G^{\prime}\left\|\boldsymbol{e}^{\prime}\right\|+R_{Y}$. Substituting in (35) gives $f(x) \leq 2 G^{\prime}\left(\left\|\boldsymbol{e}^{\prime}\right\|-x\right)+R_{Y}-\tau_{M}$ and the result follows.

Using Eq. (37) and the continuity of $f$ on $[0,+\infty[$, a direct application of the intermediate value theorem shows that the solution of $f(x)=0$ lies in the bounded interval $\left.] 0, x_{\max }\right]$. This is a useful information for solving the equation $f(x)=0$ numerically. In that regard, another useful ingredient is the derivative $f^{\prime}(x)$. A direct calculation gives:

$$
f^{\prime}(x)=-\frac{\tau_{M}}{x^{2}} \frac{R_{Y}}{\|\boldsymbol{b}\|} \frac{\boldsymbol{c}}{\|\boldsymbol{c}\|}:\left(\boldsymbol{e}_{n}^{t r}-\frac{\left(\boldsymbol{b}: \boldsymbol{e}_{n}^{t r}\right)}{\|\boldsymbol{b}\|^{2}} \boldsymbol{b}\right)-2 G^{\prime} .
$$

Remark: In the case $\tau_{M}=0$, the equation $f(x)=0$ can be solved directly. From Eqs. (33-34) we have indeed

$$
x=\left\|\boldsymbol{e}_{n}^{t r}+\frac{\boldsymbol{a}}{\|\boldsymbol{a}\|}\left(\frac{\|\boldsymbol{a}\|-R_{Y}}{2 G^{\prime}}\right)\right\|
$$

and

$$
\boldsymbol{e}_{n+1}^{t r}=\boldsymbol{e}_{n}^{t r}+\frac{\boldsymbol{a}}{\|\boldsymbol{a}\|}\left(\frac{\|\boldsymbol{a}\|-R_{Y}}{2 G^{\prime}}\right) .
$$

\subsection{Saturated phase transformation}

If the value $\boldsymbol{e}_{n+1}^{t r}$ provided by (33-34) satisfies the inequality $\left\|\boldsymbol{e}_{n+1}^{t r}\right\| \leq \varepsilon_{L}$, then the solution to problem (20) is obtained. Otherwise, we need to solve

$$
\mathbf{0}=2 G^{\prime}\left(\boldsymbol{e}_{n+1}^{t r}-\boldsymbol{e}^{\prime}\right)+\tau_{M} \frac{\boldsymbol{e}_{n+1}^{t r}}{\varepsilon_{L}}+R_{Y} \frac{\boldsymbol{e}_{n+1}^{t r}-\boldsymbol{e}_{n}^{t r}}{\left\|\boldsymbol{e}_{n+1}^{t r}-\boldsymbol{e}_{n}^{t r}\right\|}+\gamma_{n+1} \boldsymbol{e}_{n+1}^{t r}
$$

with

$$
\gamma_{n+1} \geq 0,\left\|\boldsymbol{e}_{n+1}^{t r}\right\|=\varepsilon_{L}
$$


This case corresponds to saturated phase transformation. Provided $\tau_{M} \neq$ 0 , we can use a change of variable to put (38) in the same form as (30). Introducing indeed the variable $x$ defined by

$$
\frac{\tau_{M}}{x}=\frac{\tau_{M}}{\varepsilon_{L}}+\gamma_{n+1},
$$

we can see that Eq. (38) is formally identical to Eq. (30), except that $x$ is now submitted to the constraint $0 \leq x \leq \varepsilon_{L}$. Eq. (33) still holds, but the consistency equation for determining $x$ now reads as

$$
\varepsilon_{L}=\left\|\boldsymbol{e}_{n}^{t r}+\frac{\boldsymbol{b}}{\|\boldsymbol{b}\|}\left(\frac{\|\boldsymbol{b}\|-R_{Y}}{\tau_{M} / x+2 G^{\prime}}\right)\right\| .
$$

Eq. (39) can be rewritten as:

$$
g(x)=0
$$

where

$$
g(x)=\|\boldsymbol{c}\|-\varepsilon_{L} \frac{\tau_{M}}{x}-2 G^{\prime} \varepsilon_{L}
$$

and $\boldsymbol{c}$ is defined as in (36). The equation $g(x)=0$ has a unique solution in ] $\left.0, \varepsilon_{L}\right]$. We have:

$$
g^{\prime}(x)=-\frac{\tau_{M}}{x^{2}} \frac{R_{Y}}{\|\boldsymbol{b}\|} \frac{\boldsymbol{c}}{\|\boldsymbol{c}\|}:\left(\boldsymbol{e}_{n}^{t r}-\frac{\left(\boldsymbol{b}: \boldsymbol{e}_{n}^{t r}\right)}{\|\boldsymbol{b}\|^{2}} \boldsymbol{b}\right)+\varepsilon_{L} \frac{\tau_{M}}{x^{2}} .
$$

Remark: The change of variable detailed above can be used only if $\tau_{M}>$ 0 . The case $\tau_{M}=0$ can be treated by replacing $\tau_{M} / x$ with $\gamma_{n+1}$ in the previous developments. More precisely, starting from (38) and performing manipulations similar to those used in Sect. 4.2, we obtain, in the case $\tau_{M}=0$,

$$
\boldsymbol{e}_{n+1}^{t r}=\boldsymbol{e}_{n}^{t r}+\frac{\boldsymbol{b}^{\prime}}{\left\|\boldsymbol{b}^{\prime}\right\|}\left(\frac{\left\|\boldsymbol{b}^{\prime}\right\|-R_{Y}}{\gamma_{n+1}+2 G^{\prime}}\right)
$$

where $\boldsymbol{b}^{\prime}=\boldsymbol{a}-\gamma_{n+1} \boldsymbol{e}_{n}^{t r}$. Enforcing that $\left\|\boldsymbol{e}_{n+1}^{t r}\right\|=\varepsilon_{L}$ in (41) leads to the consistency equation

$$
h\left(\gamma_{n+1}\right)=0
$$

where $h\left(\gamma_{n+1}\right)=\left\|\boldsymbol{c}^{\prime}\right\|-\varepsilon_{L} \gamma_{n+1}-2 G^{\prime} \varepsilon_{L}$ and $\boldsymbol{c}^{\prime}=2 G^{\prime} \boldsymbol{e}^{\prime}-R_{Y} \boldsymbol{b}^{\prime} /\left\|\boldsymbol{b}^{\prime}\right\|$. 


\subsection{Summary}

Algorithm 1 summarizes the proposed solution procedure. The input variables are the transformation strain $\boldsymbol{e}_{n}^{t r}$ at the previous time instant $t_{n}$ and the deviatoric strain $\boldsymbol{e}^{\prime}=G \boldsymbol{e} /(G+h / 2)$ at the current time instant $t_{n+1}$. The output variable is the transformation strain $\boldsymbol{e}_{n+1}^{t r}$ at $t_{n+1}$. The stress $\boldsymbol{\sigma}_{n+1}$ at time $t_{n+1}$ is given by

$$
\boldsymbol{\sigma}_{n+1}=\mathbb{C}:\left(\varepsilon-\boldsymbol{e}_{n+1}^{t r}\right)
$$

where $\mathbb{C}$ is the elasticity tensor. Let 1 and $\mathbb{I}$ be the second-order and fourthorder identity tensors, respectively. Setting $\mathbb{J}=(\mathbf{1} \otimes \mathbf{1}) / 3$ and $\mathbb{K}=\mathbb{I}-\mathbb{J}$, we note for later reference that

$$
\mathbb{C}=3 \kappa \mathbb{J}+2 G \mathbb{K}, \boldsymbol{\sigma}_{n+1}=\mathbb{C}: \boldsymbol{\varepsilon}-2 G \boldsymbol{e}_{n+1}^{t r}
$$

where the second equality results from the fact that $\boldsymbol{e}_{n+1}^{t r}$ is traceless.

Algorithm 1 follows a two-step procedure that is typical of return mapping strategies and similar to the elastic prediction - plastic correction decomposition used in plasticity (Simo and Hugues, 1998). Step 1 consists in checking for special cases of evolutions - namely elastic evolution or fully reversed phase transformation. This is accomplished by checking whether the input data $\left(\boldsymbol{e}_{n}^{t r}, \boldsymbol{e}^{\prime}\right)$ verify certain explicit inequalities. If those inequalities are not satisfied, then the algorithm moves to Step 2 in which a nonlinar problem is solved.

Such a two-step structure is commonly found in the algorithms for the Souza-Auricchio model, discussed in Section 1. A distinctive feature of Algorithm 1 is that the nonlinear problem involves a single scalar parameter, whereas the other mentioned algorithms use 5, 6, or 7 scalar parameters. An expected outcome is a significant gain in computational costs. It is worth highlighting that the computational cost for the factorization is $O\left(n^{3} / 3\right)$, where $n$ is the matrix size (Quarteroni, 2009), and the computation of the jacobian for a system of equations increases the computational time. Another related benefit is a gain in robustness. As mentioned earlier, the NewtonRaphson procedure - which is often used for solving nonlinear problems is known to perform very fast provided the initial guess is close enough to the solution. Failing to have a good initial guess may lead to difficulties in convergence. The issue of finding such a good initial guess simplifies considerably when the problem depends on a single scalar variable; for instance, 
a bisection method can be used, which consists only in iterative function evaluations that do not increase excessively the computational time.

Since it involves a single scalar unknown in Step 2, Algorithm 1 is similar in spirit to the radial return algorithm originally proposed by Wilkins (1964) in perfect plasticity. This is especially clear when $\tau_{M}=0$, i.e., at low temperature. In that case, there is a simple geometric interpretation of Step 2 in Algorithm 1 (see Fig. 1): Starting from the trial elastic state $\boldsymbol{a}=2 G^{\prime}\left(\boldsymbol{e}^{\prime}-\boldsymbol{e}_{n}^{t r}\right)$, a correction along the 'radial direction' $\boldsymbol{a}$ such that $\left\|2 G^{\prime}\left(\boldsymbol{e}^{\prime}-\boldsymbol{e}_{n+1}^{t r}\right)\right\|=R_{Y}$ (in the case of unsaturated phase transformation) or $\left\|\boldsymbol{e}_{n+1}^{t r}\right\|=\varepsilon_{L}$ (in the case of saturated phase transformation). The situation is more complicated when $\tau_{M}>0$. For simplicity we only discuss the case of unsaturated phase transformation. Note from (26) that the ball with center $\tau_{M} \boldsymbol{e}_{n}^{t r} /\left\|\boldsymbol{e}_{n}^{t r}\right\|$ and radius $R_{Y}$ defines the elastic domain at time $t_{n}$. If the trial elastic state $\boldsymbol{a}$ is outside of that domain, a correction is performed in such fashion that $\boldsymbol{a}_{n+1}=2 G^{\prime}\left(\boldsymbol{e}^{\prime}-\boldsymbol{e}_{n+1}^{t r}\right)$ falls on the boundary of the elasticity domain at time $t_{n+1}$, i.e., satisfies $\left\|\boldsymbol{a}_{n+1}-\tau_{M} \boldsymbol{e}_{n+1}^{t r} /\right\| \boldsymbol{e}_{n+1}^{t r}\|\|=R_{Y}$. When $\tau_{M} \neq 0$, the correction $\boldsymbol{a}_{n+1}-\boldsymbol{a}$ is no longer parallel to $\boldsymbol{a}$, as can be seen from Eq. (32). The situation is represented in Fig. 2. The increment $\boldsymbol{e}_{n+1}^{t r}-\boldsymbol{e}_{n}^{t r}$ is parallel to the correction $\boldsymbol{a}_{n+1}-\boldsymbol{a}$. Also note that $\boldsymbol{a}, \boldsymbol{a}_{n+1}$ are aligned with the center $\tau_{M} \boldsymbol{e}_{n+1}^{t r} /\left\|\boldsymbol{e}_{n+1}^{t r}\right\|$ of the elasticity domain at time $t_{n+1}$. All those geometric properties are direct consequences of Eq. (30). 


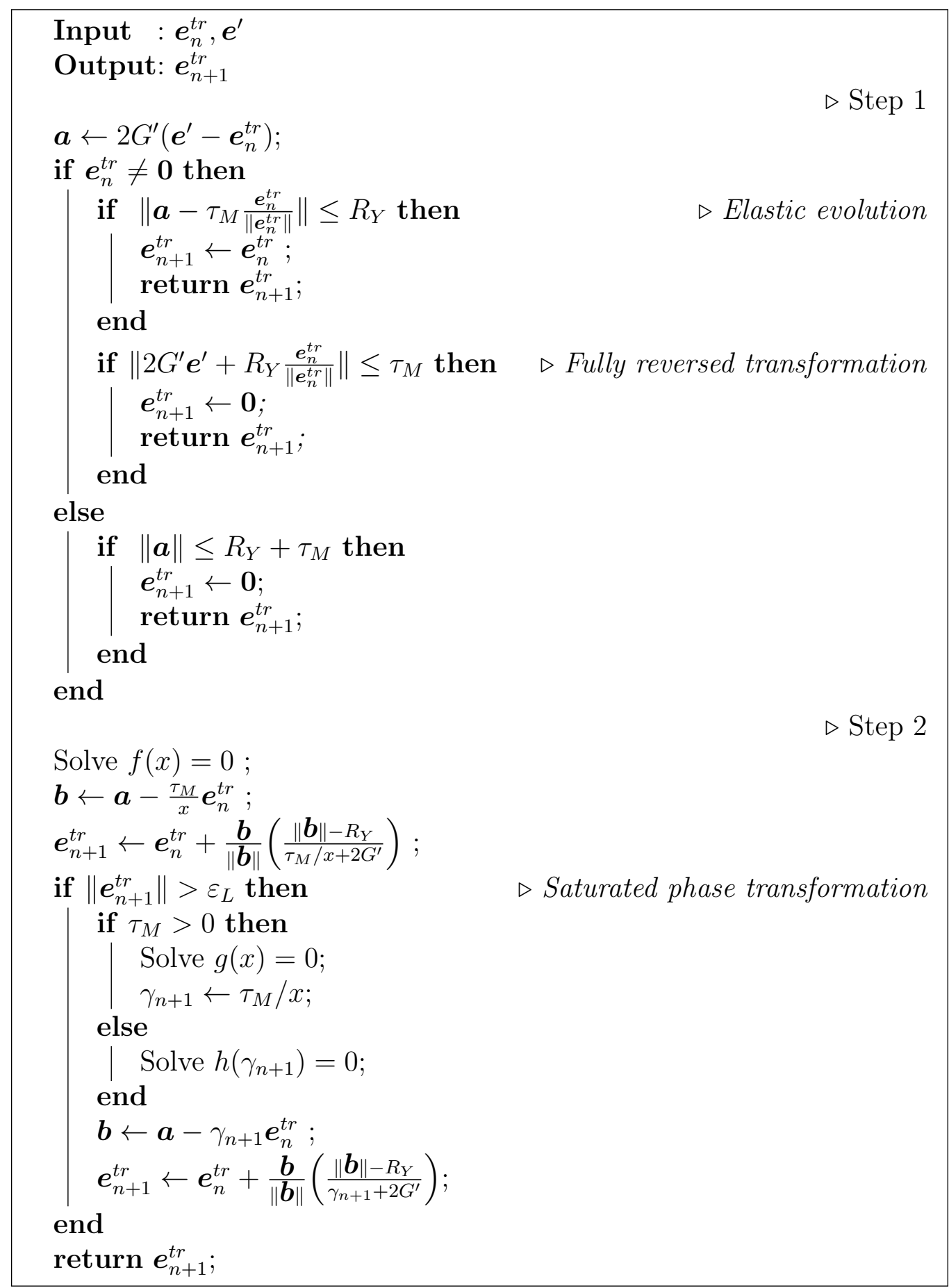

Algorithm 1: Proposed radial return algorithm for SMAs. 


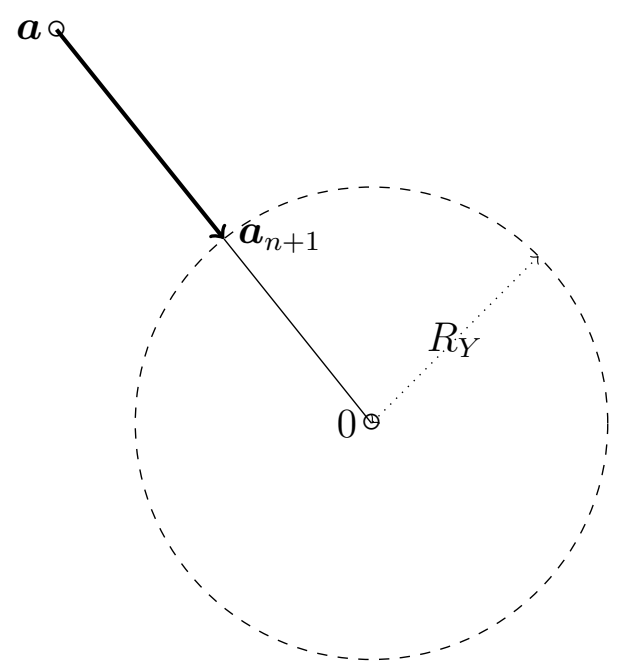

Figure 1: Radial return for $\tau_{M}=0$, case of unsaturated phase transformation.

\subsection{Tangent stiffness operator}

Using the expressions given in Sects. 4.1-4.3, the tangent stiffness operator $d \boldsymbol{\sigma}_{n+1} / d \boldsymbol{\varepsilon}$ can be calculated explicitly. In the structure of Algorithm 1 there appear 4 distinct regimes: elastic evolution $\left(\boldsymbol{e}_{n+1}^{t r}=\boldsymbol{e}_{n}^{t r}\right)$, fully reversed phase transformation $\left(\boldsymbol{e}_{n+1}^{t r}=\mathbf{0}\right)$, unsaturated phase transformation $\left(0<\left\|\boldsymbol{e}_{n+1}^{t r}\right\|<\varepsilon_{L}\right.$ with $\left.\boldsymbol{e}_{n+1}^{t r} \neq \boldsymbol{e}_{n}^{t r}\right)$ and saturated phase transformation $\left(\left\|\boldsymbol{e}_{n+1}^{t r}\right\|=\varepsilon_{L}\right.$ with $\left.\boldsymbol{e}_{n+1}^{t r} \neq \boldsymbol{e}_{n}^{t r}\right)$. The operator $d \boldsymbol{\sigma}_{n+1} / d \boldsymbol{\varepsilon}$ takes a different expressions in each one of those regimes. In the case $\boldsymbol{e}_{n+1}^{t r}=\boldsymbol{e}_{n}^{t r}$ or $\boldsymbol{e}_{n+1}^{t r}=\mathbf{0}$, we have $d \boldsymbol{\sigma}_{n+1} / d \varepsilon=\mathbb{C}$, where $\mathbb{C}$ is the elasticity tensor of the material, see Eq. (42). The expressions get more involved in the remaining cases. More precisely, we have

- in the case $0<\left\|\boldsymbol{e}_{n+1}^{t r}\right\|<\varepsilon_{L}\left(\right.$ with $\left.\boldsymbol{e}_{n+1}^{t r} \neq \boldsymbol{e}_{n}^{t r}\right)$ :

$$
\begin{array}{r}
\frac{d \boldsymbol{\sigma}_{n+1}}{d \boldsymbol{\varepsilon}}=\mathbb{C}-4 G^{2} \frac{x\left(\|\boldsymbol{b}\|-R_{Y}\right)}{\|\boldsymbol{b}\|\|\boldsymbol{c}\|}(\mathbb{K}+ \\
X_{11} \tilde{\boldsymbol{e}}_{n+1}^{t r} \otimes \tilde{\boldsymbol{e}}_{n+1}^{t r}+X_{22} \tilde{\boldsymbol{b}} \otimes \tilde{\boldsymbol{b}} \\
\left.+X_{12}\left(\tilde{\boldsymbol{e}}_{n+1}^{t r} \otimes \tilde{\boldsymbol{b}}+\tilde{\boldsymbol{b}} \otimes \tilde{\boldsymbol{e}}_{n+1}^{t r}\right)\right)
\end{array}
$$

- in the case $\left\|\boldsymbol{e}_{n+1}^{t r}\right\|=\varepsilon_{L}\left(\right.$ with $\left.\boldsymbol{e}_{n+1}^{t r} \neq \boldsymbol{e}_{n}^{t r}\right)$ :

$$
\frac{d \boldsymbol{\sigma}_{n+1}}{d \boldsymbol{\varepsilon}}=\mathbb{C}-4 G^{2} \frac{\varepsilon_{L}\left(\|\boldsymbol{b}\|-R_{Y}\right)}{\|\boldsymbol{b}\|\|\boldsymbol{c}\|}\left(\mathbb{K}-\tilde{\boldsymbol{e}}_{n+1}^{t r} \otimes \tilde{\boldsymbol{e}}_{n+1}^{t r}+\frac{R_{Y}}{\|\boldsymbol{b}\|-R_{Y}\left(1-\alpha^{2}\right)} \boldsymbol{b}_{\perp} \otimes \boldsymbol{b}_{\perp}\right)
$$




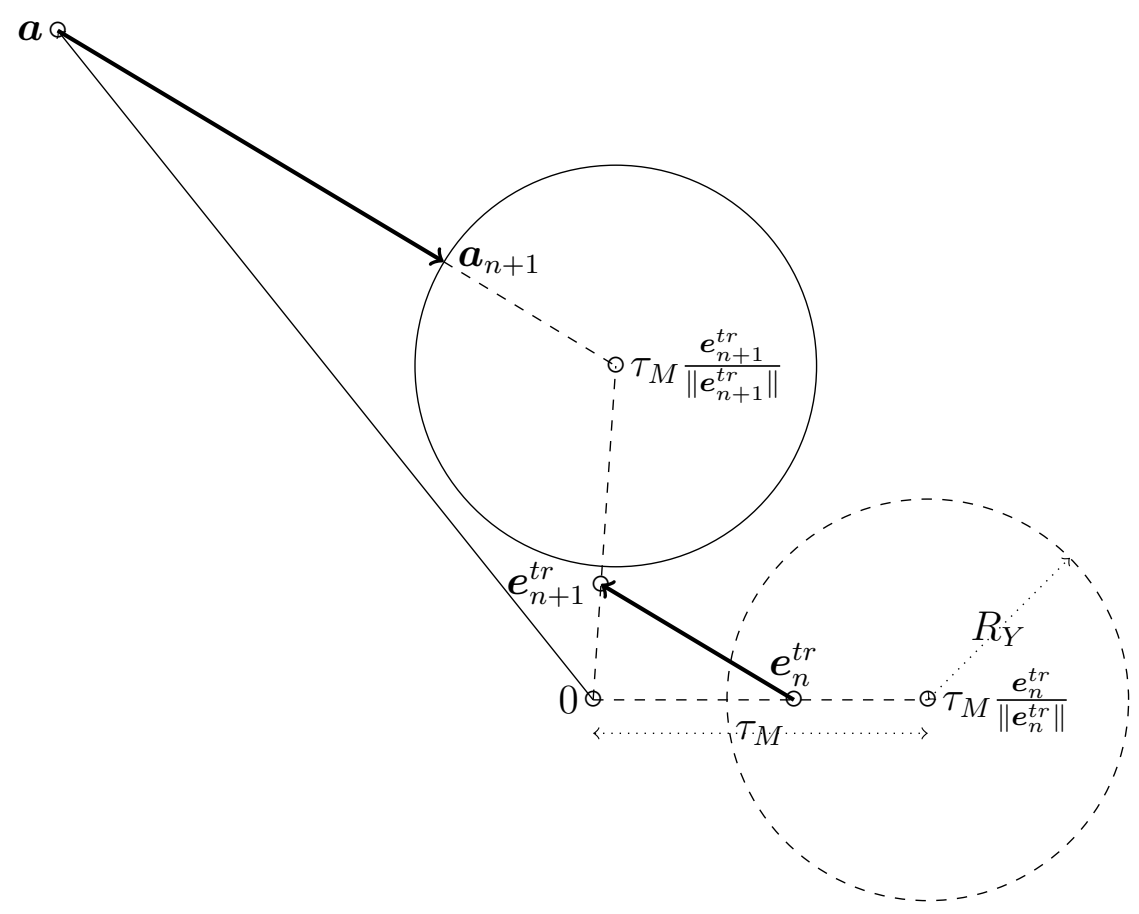

Figure 2: Radial return for $\tau_{M}>0$, case of unsaturated phase transformation. $\mathbb{C}$.

Recall that, in the case $\left\|\boldsymbol{e}_{n+1}^{t r}\right\|=\varepsilon_{L}$ with $\boldsymbol{e}_{n+1}^{t r}=\boldsymbol{e}_{n}^{t r}$, we have $d \boldsymbol{\sigma}_{n+1} / d \boldsymbol{\varepsilon}=$

In Eqs. (43-44), $\tilde{\boldsymbol{e}}_{n+1}^{t r}$ and $\tilde{\boldsymbol{b}}$ are the unit tensors defined as $\tilde{\boldsymbol{e}}_{n+1}^{t r}=$ $\boldsymbol{e}_{n+1}^{t r} /\left\|\boldsymbol{e}_{n+1}^{t r}\right\|$ and $\tilde{\boldsymbol{b}}=\boldsymbol{b} /\|\boldsymbol{b}\|$, respectively. The tensor $\boldsymbol{b}_{\perp}$ is the projection of $\tilde{\boldsymbol{b}}$ on the orthogonal of $\boldsymbol{e}_{n+1}^{t r}$, i.e., $\boldsymbol{b}_{\perp}=\tilde{\boldsymbol{b}}-\alpha \tilde{\boldsymbol{e}}_{n+1}^{t r}$ with $\alpha=\tilde{\boldsymbol{e}}_{n+1}^{t r}: \tilde{\boldsymbol{b}}$. The scalars $X_{11}, X_{22}, X_{12}$ in (43) are defined by

$$
X_{11}=\frac{\left(\|\boldsymbol{b}\|-R_{Y}\right) \tau_{M}}{X}, X_{22}=\frac{R_{Y}}{X}\left(\frac{\|\boldsymbol{b}\|\|\boldsymbol{c}\|}{\|\boldsymbol{b}\|-R_{Y}}-\tau_{M}\right), X_{12}=\frac{\alpha R_{Y} \tau_{M}}{X},
$$

where $X=\|\boldsymbol{b}\|\left(\|\boldsymbol{c}\|-\tau_{M}\right)+\left(1-\alpha^{2}\right) R_{Y} \tau_{M}$.

The derivation of Eqs. (43-44) is detailed in Appendix B. We emphasize the fact that expressions (43-44) are fully explicit and do not involve any matrix inversion, thus making for an efficient numerical computation of the tangent stiffness operator. 


\section{Numerical simulations}

This section presents several numerical simulations to show the performance of the proposed radial return algorithm. The conducted finite element analyses include simple uniaxial and biaxial tests as well as two complex simulations, reproducing both pseudoelasticity and shape memory effect. The algorithm proposed in Auricchio and Petrini (2004) (in the following, denoted as classical for simplicity) has been implemented to provide a comparison. The adopted material parameters are reported in Table 1.

The proposed algorithm has been implemented within a user-defined material subroutine (UMAT) of the finite element software ABAQUS/Standard (Abaqus, 2010). We use the package AceGen (Korelc, 2002) of the symbolic software Mathematica to generate the UMAT, according to the methodology proposed in Boatti et al. (2016).

Table 1: Material parameters adopted in all the numerical simulations (Auricchio et al., 2009a). Regularization $\delta$ is used only in the classical algorithm.

\begin{tabular}{lcl}
\hline Symbol & Value & Unit \\
\hline$E$ & 53,000 & $\mathrm{MPa}$ \\
$\nu$ & 0.33 & - \\
$h$ & 1,000 & $\mathrm{MPa}$ \\
$\varepsilon_{L}$ & 0.056 & - \\
$T^{*}$ & 243 & $\mathrm{~K}$ \\
$\beta$ & 6.1 & $\mathrm{MPa} / \mathrm{K}$ \\
$R_{Y}$ & 100 & $\mathrm{MPa}$ \\
$\delta$ & $10^{-6}$ & - \\
\hline
\end{tabular}

\subsection{Isothermal tests}

We start by considering several three-dimensional isothermal tests.

First, we simulate uniaxial tension-compression tests on a single 8-node hexahedral element, under displacement control and prescribed homogeneous temperature. Figure 3(a) shows the applied boundary conditions. In particular, a displacement is applied and increased up to $0.07 \mathrm{~mm}$ and three temperature values of 350,255 , and $200 \mathrm{~K}$ are considered.

Figures 4(a), 4(c), and 4(e) reports the stress-strain curves predicted by the proposed algorithm for time steps, respectively, of $0.002,0.02$, and 0.2 s, corresponding to 1000, 100, and 10 increments. The curves demonstrate 


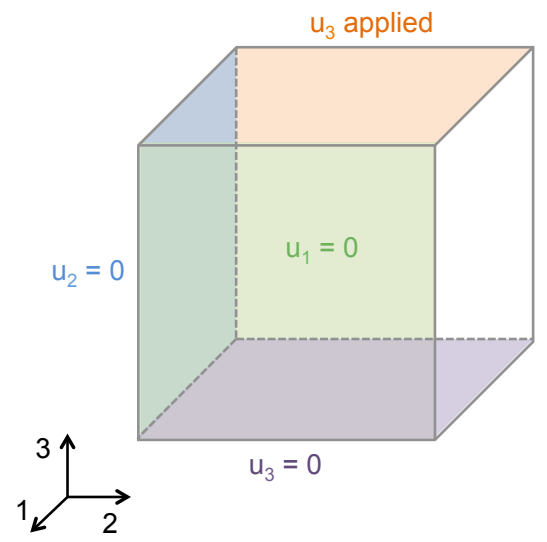

(a)

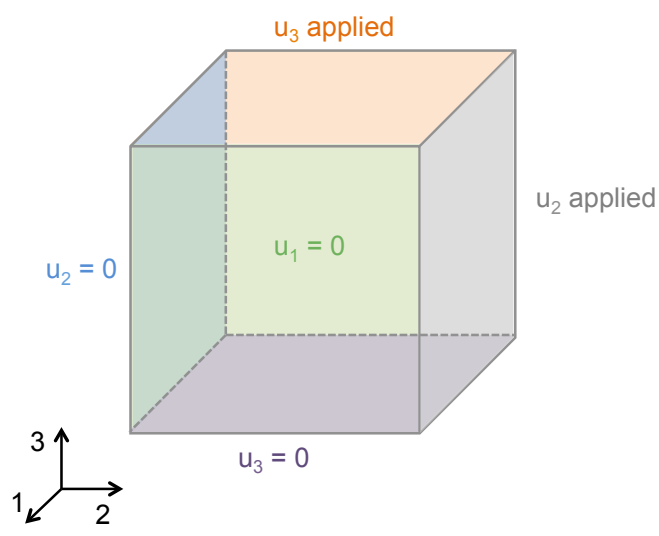

(b)

Figure 3: Applied boundary conditions for the (a) uniaxial tension-compression and (b) biaxial tests under displacement-control and constant prescribed temperature.

the robustness of the proposed solution algorithm. In order to verify model response, Figures 4(b), 4(d), and 4(f) compares the curves obtained with the proposed and the classical algorithm for 350,255 , and $200 \mathrm{~K}$, respectively. A qualitative agreement between the two algorithms is evident; however, the predicted responses differ at the beginning of the forward phase transformation and at the end of the reverse phase transformation. This is due to the presence of the regularized parameter $\delta$ in the classical algorithm, which affects model response, especially when the norm of the transformation strain $\left\|\boldsymbol{e}^{t r}\right\|$ tends to zero.

As a further comparison, we consider the uniaxial tensile test at constant temperature of $350 \mathrm{~K}$ and time step of $0.02 \mathrm{~s}$. Figures 5(a) and (b) report the number of iterations required by the (local) Newton-Raphson scheme in the unsaturated and saturated cases (see Sections 4.2 and 4.3) versus the controlled strain component $\epsilon_{33}$, for the two algorithms during loading and unloading, respectively. The local iteration number is the same for all the integration points. The values $\epsilon_{33}=6.72 \cdot 10^{-2}$ and $\epsilon_{33}=7.00 \cdot 10^{-2}$ correspond to the saturated case for both the algorithms. Moreover, it is noticed that the classical algorithm starts the phase transformation earlier during loading (i.e., at $\epsilon_{33}=2.80 \cdot 10^{-3}$ ) and ends the phase transformation later during unloading (i.e., again at $\epsilon_{33}=2.80 \cdot 10^{-3}$ ). This increases the computational time for the classical algorithm, since it has to solve a nonlinear system of equations, while the proposed algorithm has to perform an elastic check and 
the iteration number is equal to zero in Figure 5. It can be noticed that the number of iterations is higher for the classical algorithm in the unsaturated case, thus resulting in longer computational times.

We now consider biaxial tests on a single 8-node hexahedral element, under displacement control and prescribed homogeneous temperature. Figure 3(b) shows the applied boundary conditions. The loading consists of a butterfly-shaped history at a constant temperature of $400 \mathrm{~K}$. A maximum displacement of $0.1 \mathrm{~mm}$ is applied, as shown in Figure 6. To demonstrate the robustness of the proposed solution algorithm, Figure 7(a) reports the curves in terms of the non-zero stress components predicted by the proposed algorithm for time steps, respectively, of $0.004,0.04$, and $0.4 \mathrm{~s}$, corresponding to 1000, 100, and 10 increments. Figure 7(b) shows a good matching between the curves obtained with the proposed and the classical algorithm. Differences between the two algorithms are clearer in Figure 8, where the transformation strain norm $\left\|\boldsymbol{e}^{t r}\right\|$ is plotted. The classical algorithm does not reach zero values of the norm under zero stress and strain states (see time instants of 2 and $4 \mathrm{~s}$ ).

Finally, we conclude this subsection with the simulation of a square plate with a circular hole, subjected to displacement-control loading at a constant high temperature. The plate has an edge length of $100 \mathrm{~mm}$, a thickness of $2 \mathrm{~mm}$, and a central circular hole of radius $10 \mathrm{~mm}$. According to the symmetry of the problem, we model only one quarter of the plate by applying appropriate boundary conditions. The mesh is composed of 8,712 8-node hexahedral elements and 13,467 nodes, as shown in Figure 9; a mesh refinement has been performed to choose the appropriate mesh. A displacement $u_{Y}$ is applied at the top side of the plate at a temperature of $400 \mathrm{~K}$ (see Figure 9). A maximum value of $5 \mathrm{~mm}$ is applied and then set back to zero, as reported in Figure 10(a). The simulation is performed using variable time steps between $10^{-6}$ and $0.1 \mathrm{~s}$.

The deformed mesh under the maximum displacement is reported in Figure 10(b). Figure 11 shows the reaction force of the bottom side of the plate versus the applied displacement diagram; a comparison with the results obtained with the classical algorithm is also provided. As it can be observed, the pseudoelastic response of the plate is correctly reproduced by both the algorithms, but differences are evident mostly at the beginning of forward transformation and at the end of reverse transformation, i.e., around displacements of 0.2 and $1.3 \mathrm{~mm}$. This is clear from Figure 12 which represents the contour plot of the norm of the transformation strain $\left\|e^{t r}\right\|$ at different 


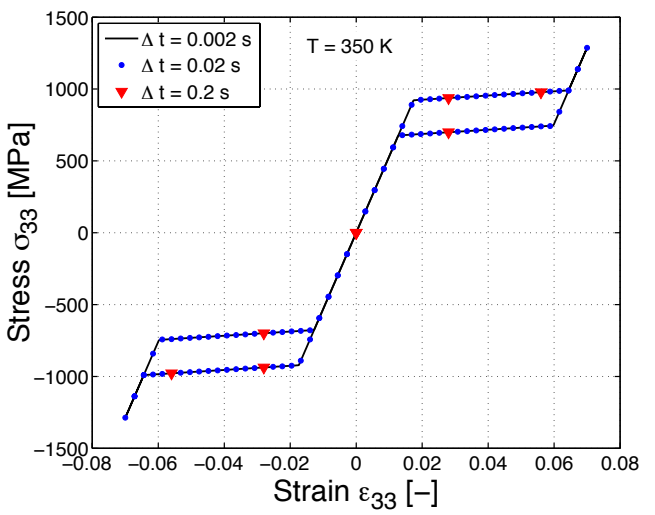

(a)

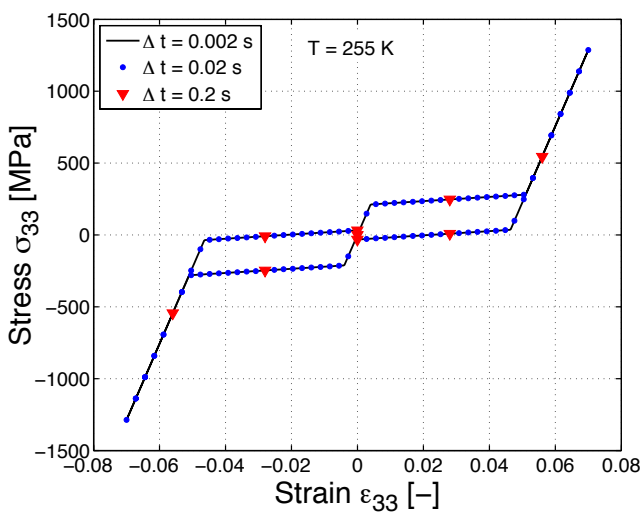

(c)

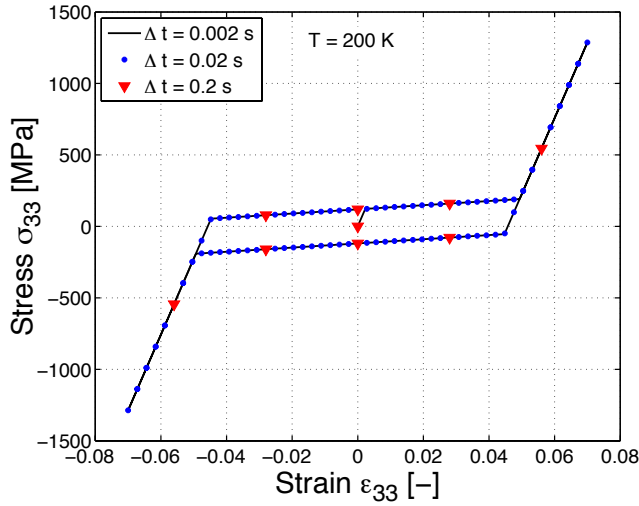

(e)

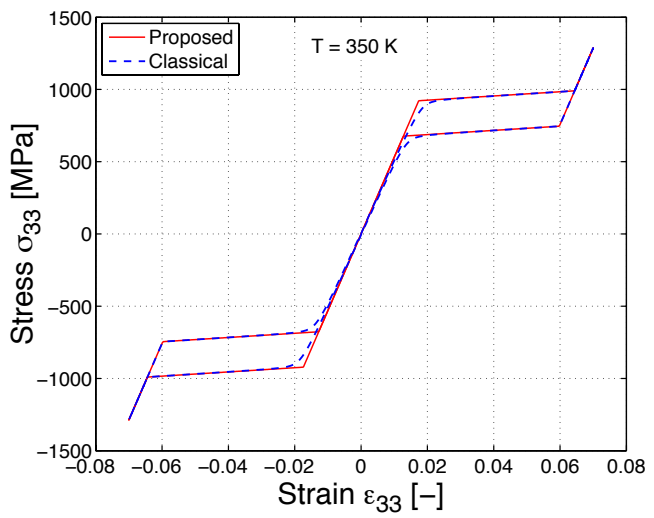

(b)

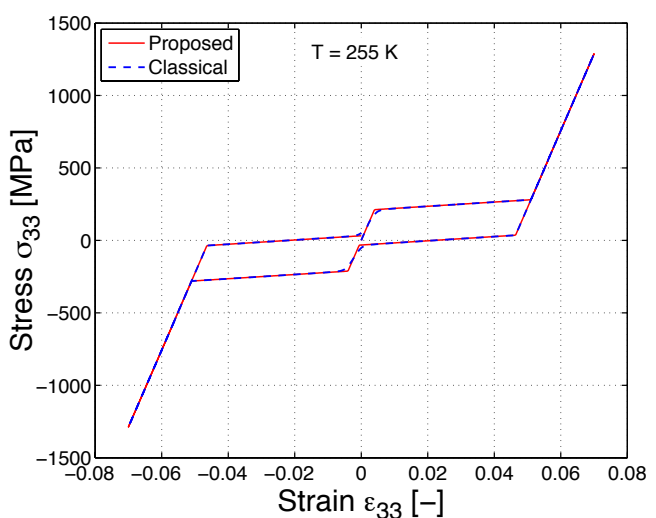

(d)

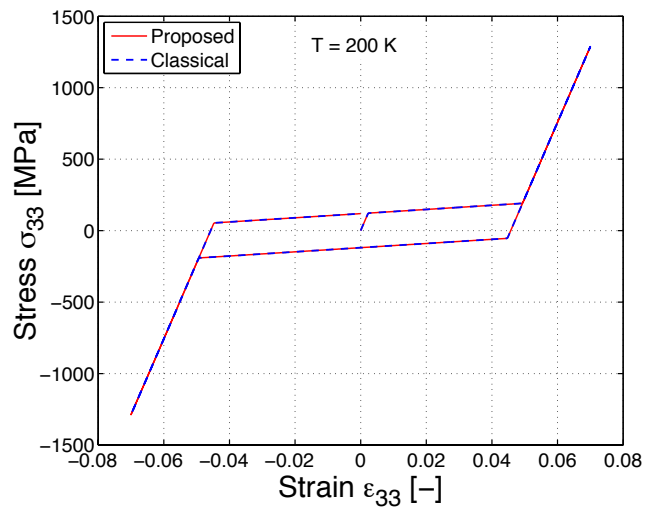

(f)

Figure 4: Uniaxial tests under displacement-control and constant prescribed temperatures of 350, 255, and 200 K. (a)-(c)-(e) Stress-strain curves obtained with the proposed algorithm for different time steps $\Delta t$. (b)-(d)-(f) Comparison between the proposed and the classical algorithm. 


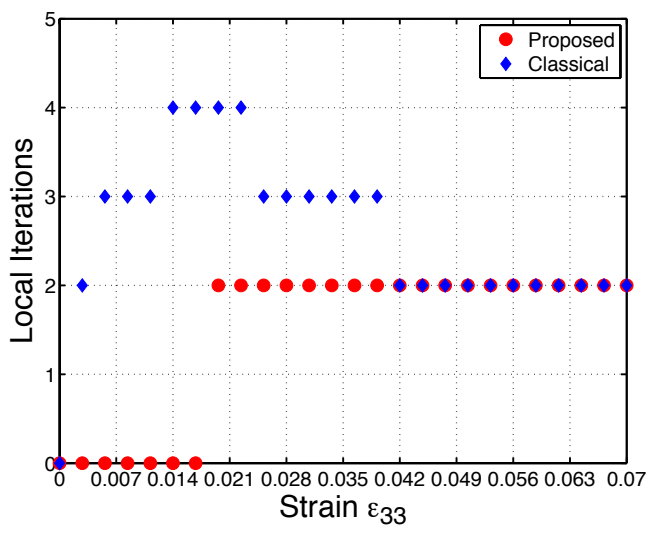

(a)

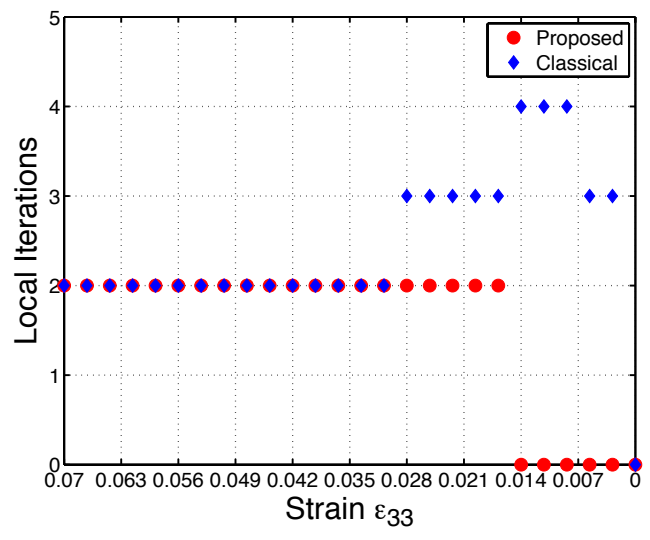

(b)

Figure 5: Uniaxial test under displacement-control and constant prescribed temperatures of $350 \mathrm{~K}$. Comparison between the number of iterations required by the (local) NewtonRaphson scheme at the integration point level in the unsaturated and saturated case for the classical and proposed algorithm. (a) Loading and (b) unloading cases.

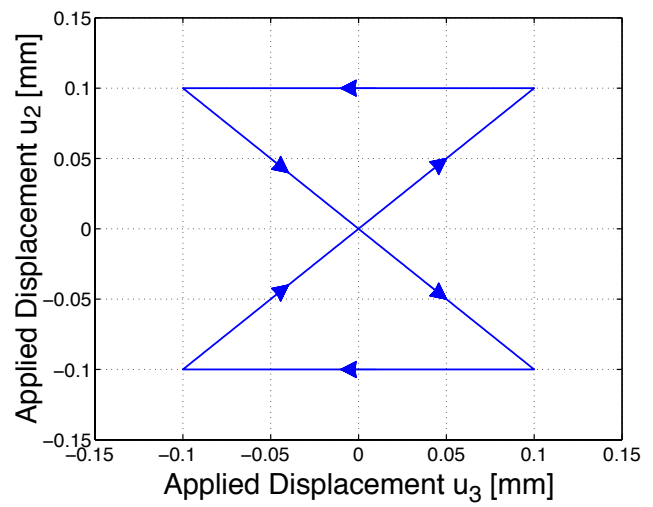

Figure 6: Biaxial test under displacement-control and constant prescribed temperature of $400 \mathrm{~K}$. The applied butterfly-shaped history is represented. 


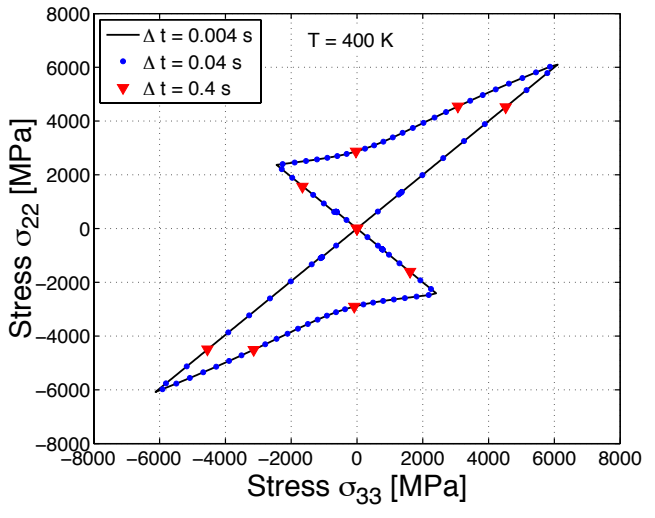

(a)

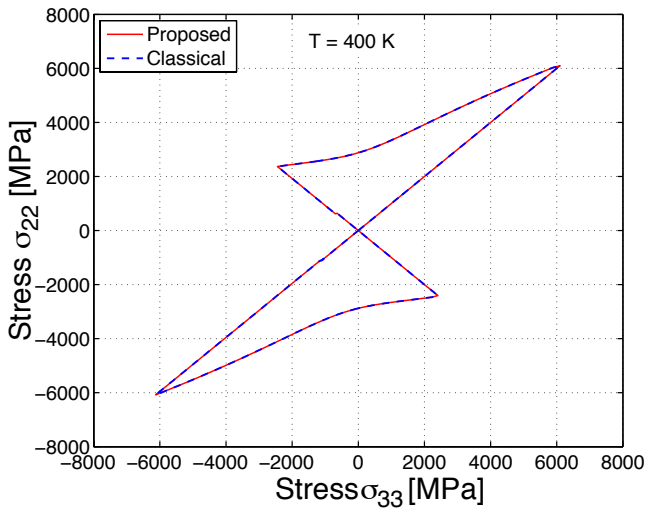

(b)

Figure 7: Biaxial test under displacement-control and constant prescribed temperature of $400 \mathrm{~K}$. Curves in terms of the non-zero stress components. (a) Results for the proposed algorithm for different time steps $\Delta t$. (b) Comparison between the proposed and the classical algorithm.

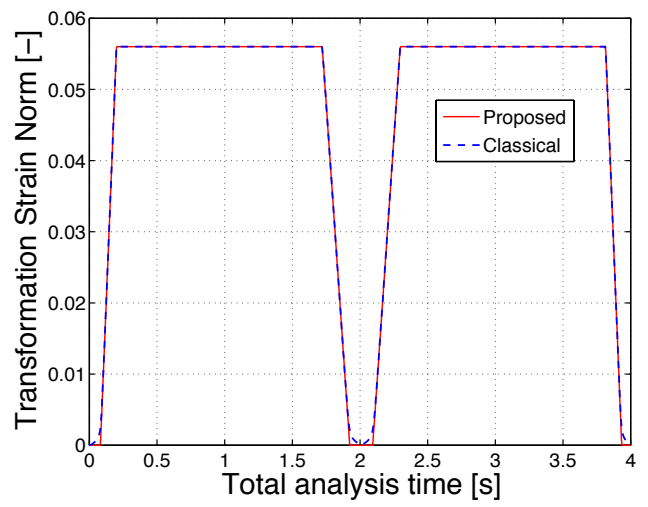

Figure 8: Biaxial test under displacement-control and constant prescribed temperature of $400 \mathrm{~K}$. Trend of the norm of the transformation strain $\left\|e^{t r}\right\|$ obtained with the proposed and classical algorithm. 


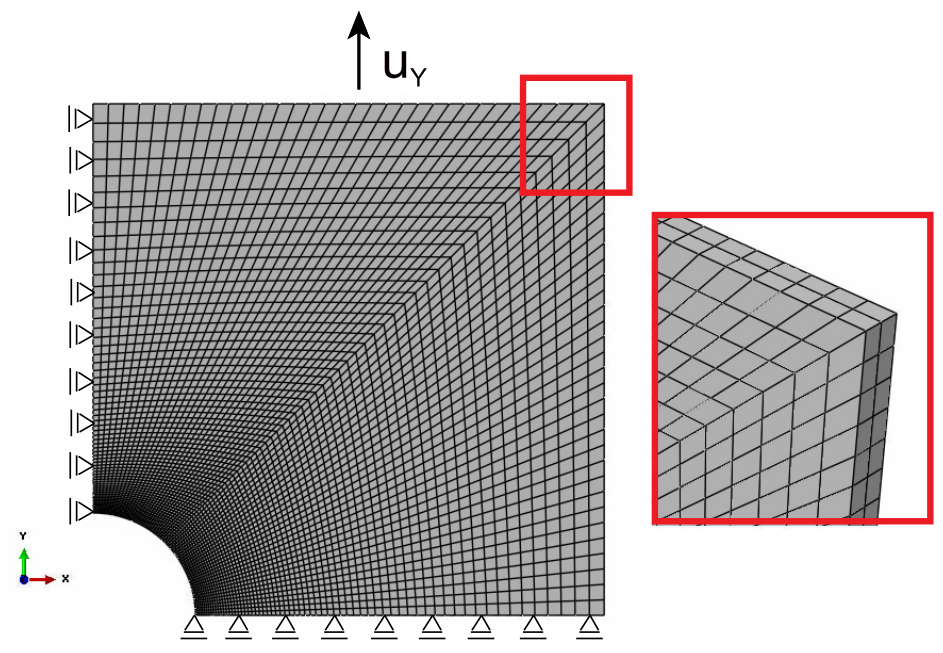

Figure 9: Pseudoelastic test on a square plate with a circular hole. Adopted mesh and applied boundary conditions.

time instants. Main discrepancies in the distribution are evident at the beginning of the analysis at $0.125 \mathrm{~s}$ and corresponding displacement of 1.25 $\mathrm{mm}$.

To assess the computational efficiency of the proposed algorithm, Figures 13(a) and 13(b) report, respectively, the loading time increments and the total global iterations during the analysis. The results are compared to those obtained with the classical algorithm. As it can be observed, the classical algorithm requires smaller time increments to converge, especially in correspondence of phase transformation. On the contrary, the proposed algorithm needs a larger number of iterations; this can be due to the use of larger time increments. Table 2 reports the total increments to complete the analysis for the two algorithms; it is worth to highlight the lower number of time increments for the proposed solution scheme. As a further comparison, we also report the wall-clock times necessary to complete the analysis in Table 3. The values clearly show the lower times required by the proposed algorithm.

\subsection{Thermal-cycling tests}

We now consider several three-dimensional thermal-cycling tests.

First we reproduce the shape memory effect through a uniaxial test on a single 8-node hexahedral element under force-control and constant low temperature, followed by a heating cycle. The applied boundary conditions 


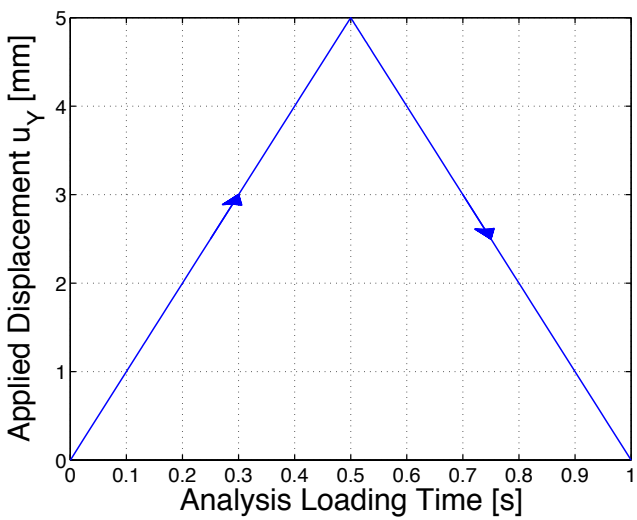

(a)

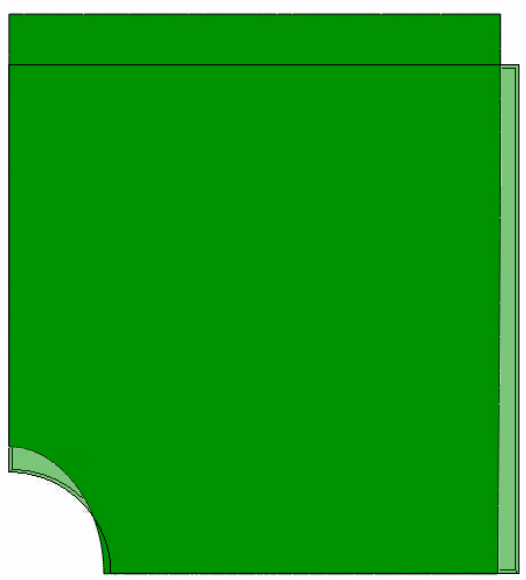

(b)

Figure 10: Pseudoelastic test on a square plate with a circular hole. (a) Loading history in terms of the applied displacement $u_{Y}$ and (b) deformed mesh at the maximum applied displacement.

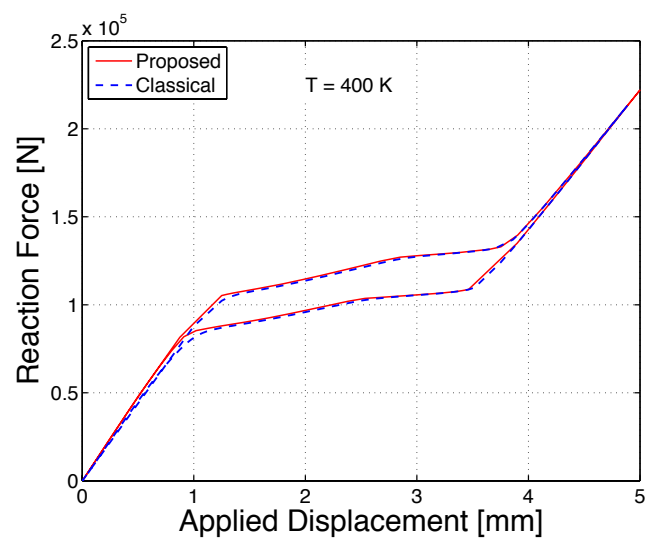

Figure 11: Pseudoelastic test on a square plate with a circular hole. Curves in terms of reaction force of the bottom side of the plate and applied displacement. Comparison between the proposed and the classical algorithm.

are those considered for the tension-compression uniaxial tests (see Figure $3(\mathrm{a}))$, except that a force is now applied on the top surface of the element. Initially, a temperature of $200 \mathrm{~K}$ is prescribed and a force of $400 \mathrm{~N}$ is applied and then set back to zero. Then, the temperature is increased up to $400 \mathrm{~K}$ at zero stress. Figure 14 reports the stress-strain-temperature curves predicted 


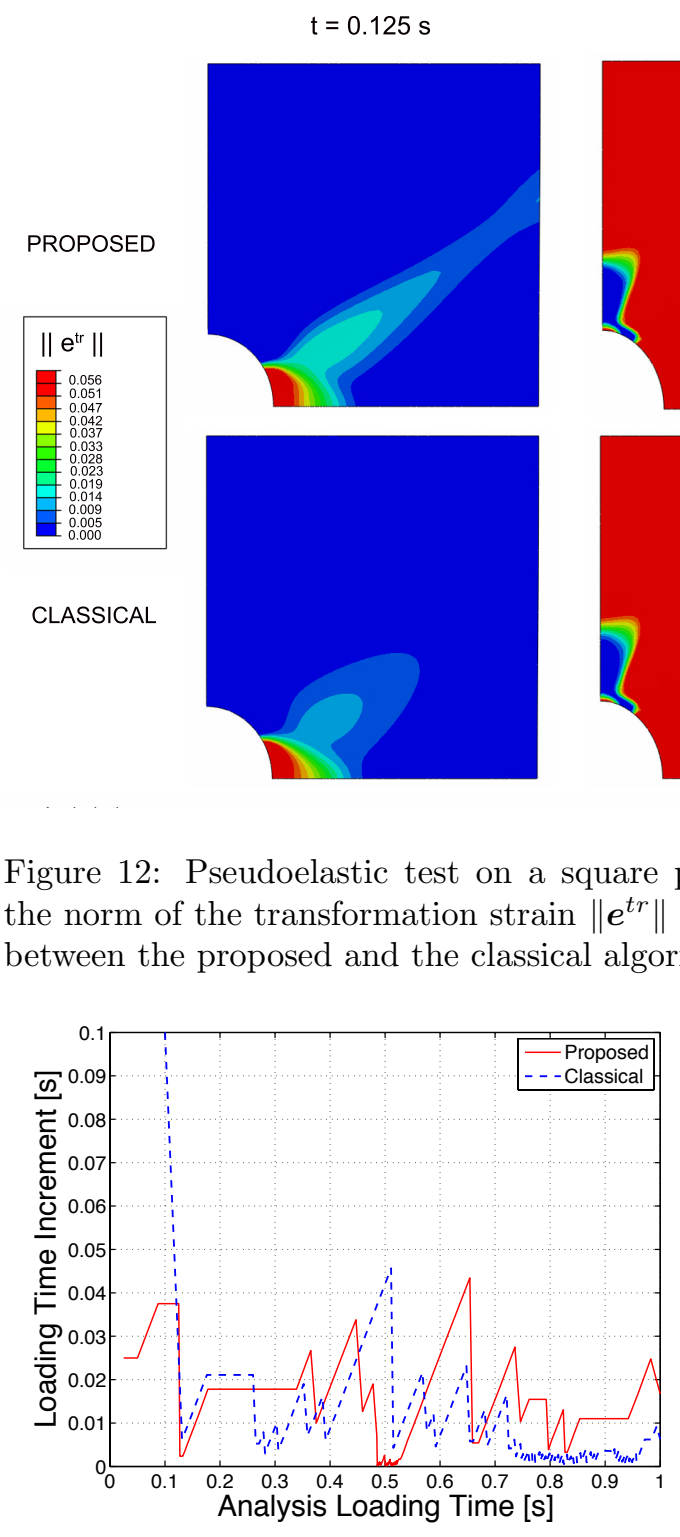

(a)

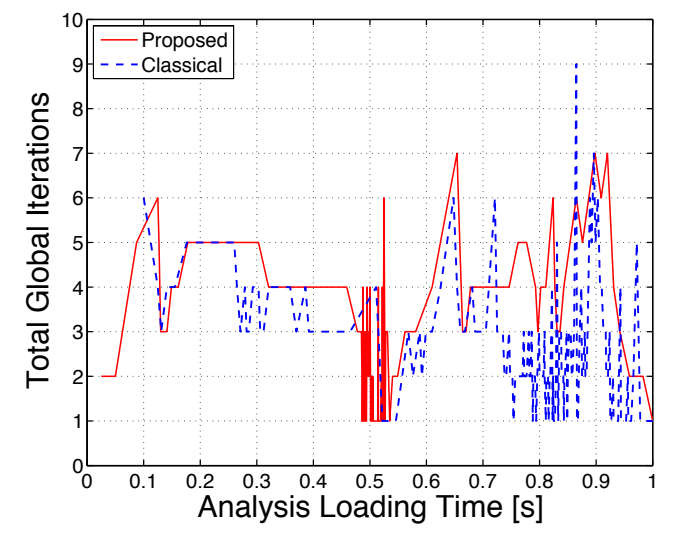

(b)

Figure 13: Pseudoelastic test on a square plate with a circular hole. Trends of (a) the loading time increments and (b) the total global iterations during the analysis. Comparison between the proposed and the classical algorithm. 
Table 2: Total time increments to complete the analysis. Comparison between the proposed and the classical algorithm.

\begin{tabular}{clc}
\hline Analysis & Proposed & Classical \\
\hline Pseudoelastic plate with hole & 128 & 174 \\
Spring actuator & 230 & 812 \\
\hline
\end{tabular}

Table 3: Wall-clock times [s] to complete the performed analyses with one CPU. Comparison between the proposed and the classical algorithm.

\begin{tabular}{clc}
\hline Analysis & Proposed & Classical \\
\hline Pseudoelastic plate with hole & 2781 & 3923 \\
Spring actuator & 3507 & 15053 \\
\hline
\end{tabular}

by the proposed algorithm for time steps, respectively, of $0.003,0.03$, and 0.3 s, corresponding to 1000, 100, and 10 increments. Figures $15(\mathrm{a})$ and $15(\mathrm{~b})$ compares the curves obtained with the proposed and the classical algorithm. The differences are evident at around $260 \mathrm{~K}$, when phase transformation starts.

We now simulate a uniaxial test on a 8-node hexahedral element under force-control and constant high temperature, followed by a thermal cycle. Initially, a temperature of $303 \mathrm{~K}$ is prescribed and a force of $300 \mathrm{~N}$ is applied and kept constant. Then, the temperature is decreased up to $121.2 \mathrm{~K}$ and finally increased up to $454.5 \mathrm{~K}$. Figure 16(a) reports the strain-temperature curves predicted by the proposed algorithm for time steps, respectively, of $0.001,0.01$, and $0.1 \mathrm{~s}$, corresponding to 17000, 1700, and 170 increments. Figures 16(b) compares the curves obtained with the proposed and the classical algorithm.

Finally, we conclude with the simulation of a helical spring actuator, subjected first to a tensile force at constant high temperature and then to a thermal cycling at constant applied force. The spring has a wire diameter of $1.0 \mathrm{~mm}$, an external coil diameter of $6.0 \mathrm{~mm}$, a pitch of $2.5 \mathrm{~mm}, 2$ active coils, and an initial length of $5.0 \mathrm{~mm}$. The mesh is composed of 37128 node hexahedral elements and 4486 nodes, as shown in Figure17(a); a mesh refinement has been performed to choose the appropriate mesh. An axial tensile force $F$ of $20 \mathrm{~N}$ is applied at one section of the spring at a temperature of $423 \mathrm{~K}$, while the other section is fixed (see Figure 17(a)). All the nodes of the section where the force is applied are constrained against the two translations in the directions orthogonal to the axial one. A thermal cycling 


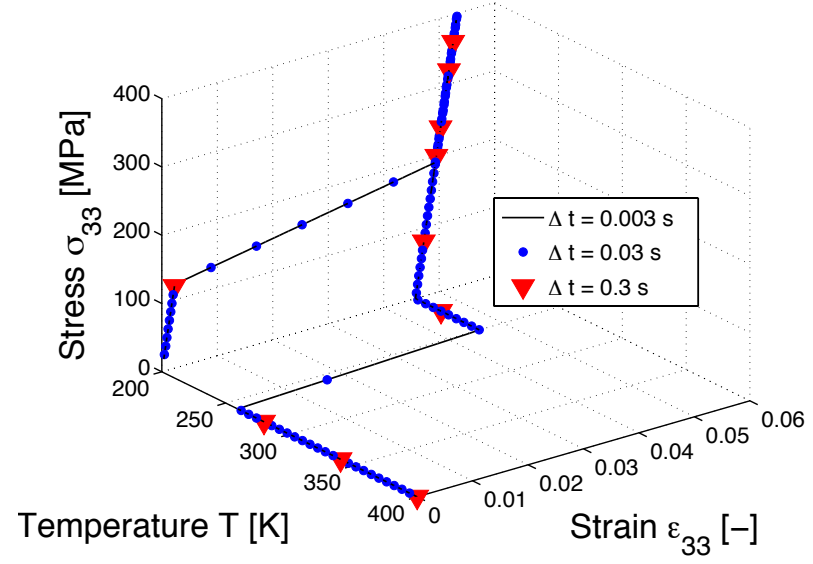

Figure 14: Uniaxial test under force-control and constant low temperature, followed by a heating cycle. Stress-strain-temperature curves obtained with the proposed algorithm for different time steps $\Delta t$.

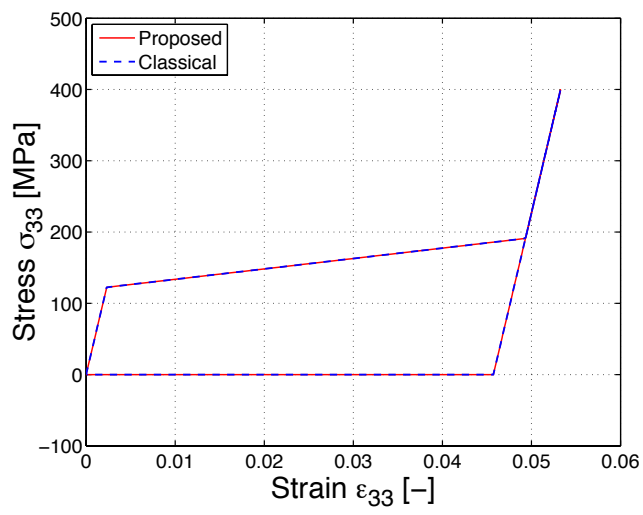

(a)

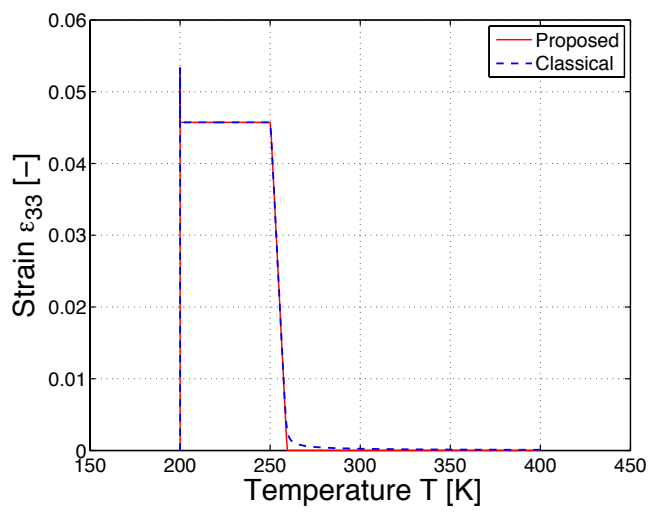

(b)

Figure 15: Uniaxial test under force-control and constant low temperature, followed by a heating cycle. Comparison between the proposed and the classical algorithm. (a) Stressstrain and (b) strain-temperature curves. 


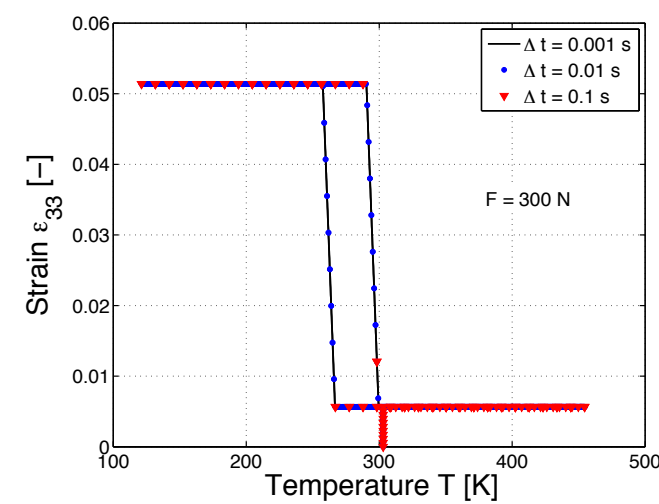

(a)

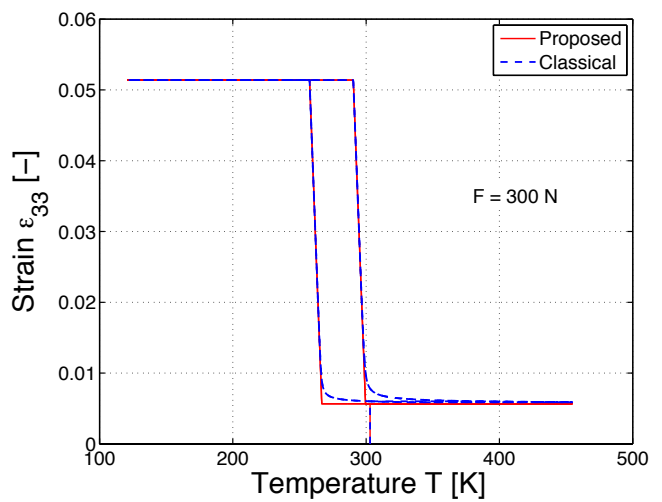

(b)

Figure 16: Strain-temperature curves for a uniaxial test with force control and at constant high temperature, followed by a thermal cycle. (a) Results of the proposed algorithm for different loading time increments $\Delta t$. (b) Comparison between the proposed and the classical algorithm.

is applied at constant force $F$, by cooling the spring up to $169.2 \mathrm{~K}$ and then heating up to the initial temperature (see Figure 18(a)). The simulation is performed using variable time step increments between $10^{-6}$ and $0.01 \mathrm{~s}$. The deformed mesh at $169.2 \mathrm{~K}$ is reported in Figure 17(b), while Figure 12 represents the related contour plot of the norm of the transformation strain $\left\|e^{t r}\right\|$.

Figure 18(b) shows the displacement of the free end of the spring versus temperature; a comparison with the results obtained with the classical algorithm is also provided. As it can be observed, the two models present differences at the beginning of forward transformation and at the end of reverse transformation.

To assess the computational efficiency of the proposed algorithm, Figures 20(a) and 20(b) report, respectively, the loading time increments and the total global iterations during the analysis. The results are compared to those obtained with the classical algorithm. As it can be observed, the classical algorithm requires smaller time increments to converge, especially in correspondence of phase transformation. On the contrary, the proposed algorithm proceeds with a larger number of iterations; this is attributed to the use of larger time increments. Table 2 reports the total increments to complete the analysis for the two formulations; it is worth to highlight the lower number of time increments for the proposed solution scheme. To provide a further 


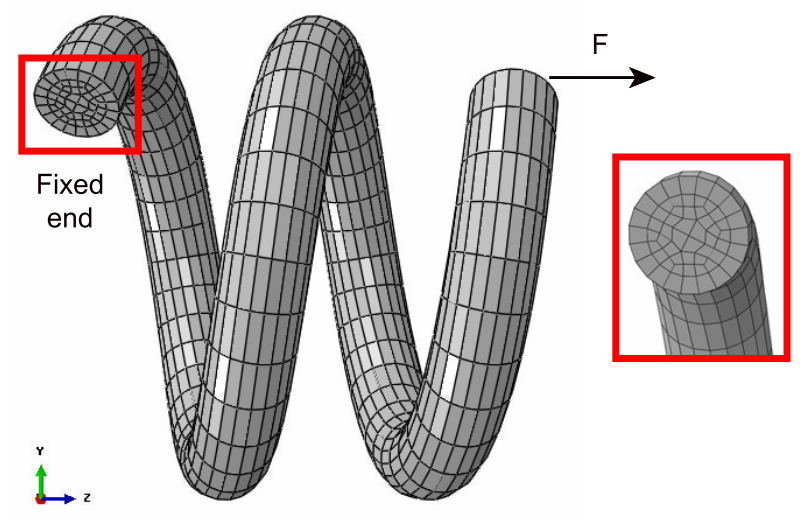

(a)

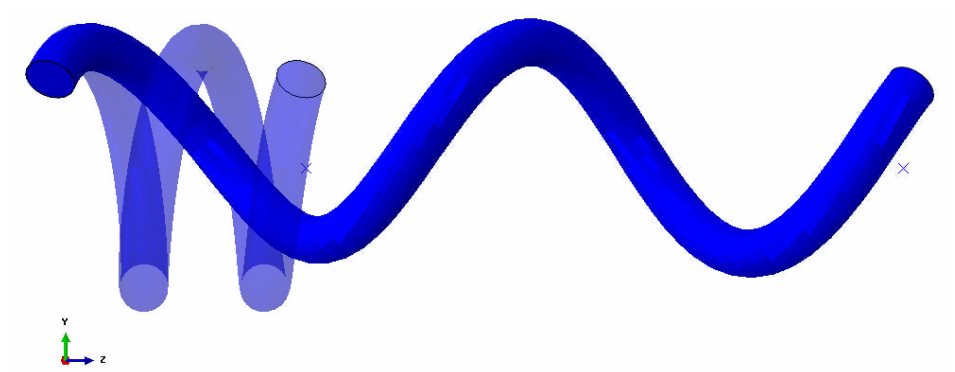

(b)

Figure 17: Thermal cycling at constant tensile axial force on a helical spring. (a) Adopted mesh and applied boundary conditions. (b) Deformed spring after cooling at $169.2 \mathrm{~K}$ and constant force of $20 \mathrm{~N}$.

comparison, we also report the wall-clock time necessary to complete the analysis in Table 3, which is considerably lower (about 1/4) for the proposed algorithm.

\section{Conclusions}

This paper has presented a new algorithmic scheme for the Souza-Auricchio model. The main idea relies in the exploitation of the variational structure of model equations, based on an incremental energy minimization approach. This results in a nonsmooth optimization problem which, using convex analysis, can be solved without any kind of regularization. This is a welcome 


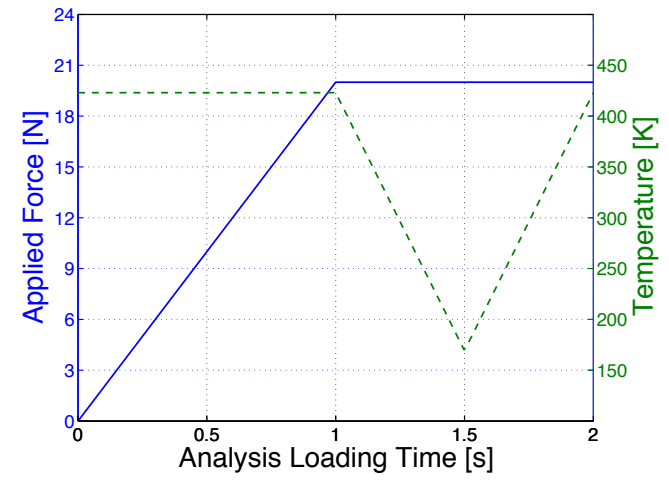

(a)

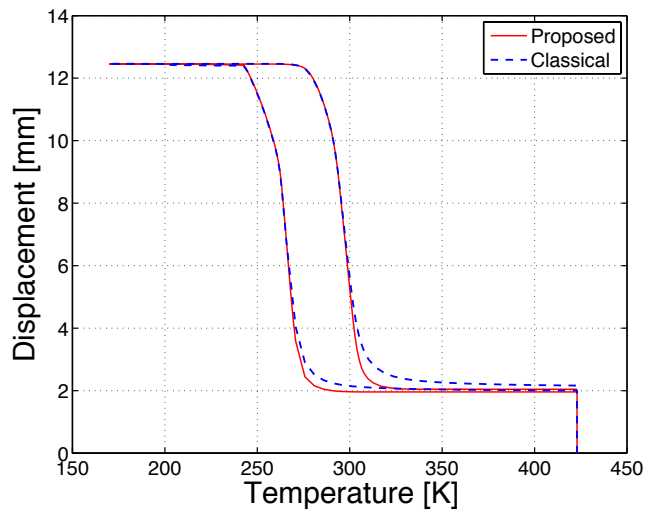

(b)

Figure 18: Thermal cycling at constant tensile axial force on a helical spring. (a) Loading history. (b) Curves in terms of displacement of the free end of the spring and prescribed temperature. Comparison between the proposed and the classical algorithm.

feature in practice: as illustrated in Section 5, adding a regularization biases the structural response, especially at the onset of phase transformation.

The proposed approach leads to a radial return scheme that is simple to implement. That scheme has the key feature of being based on the solution of a single scalar equation. The presented approach has been verified to be efficient in practice through several numerical finite element simulations, leading to low computational costs. Such an advantage can be exploited for the computer-based design of complex SMA-based devices.

We note that the proposed approach can be adapted to SMA constitutive models involving tensorial internal variables and constraints. On a related note, the easy implementation offers the possibility of extending it to more complex models, e.g., (Auricchio et al., 2007).

In this paper, thermal equilibrium was assumed to hold at each time, so that the temperature was acting as a given external parameter. That assumption is valid for small loading rates. When the loading rate increases, thermomechanical effects - most notably through latent heat and thermal diffusion effects - may become significant. Handling such effects can be achieved by combining the proposed approach with incremental variational principles proposed by Peigney and Seguin (2013) for coupled thermomechanical problems. 


\section{PROPOSED}

CLASSICAL
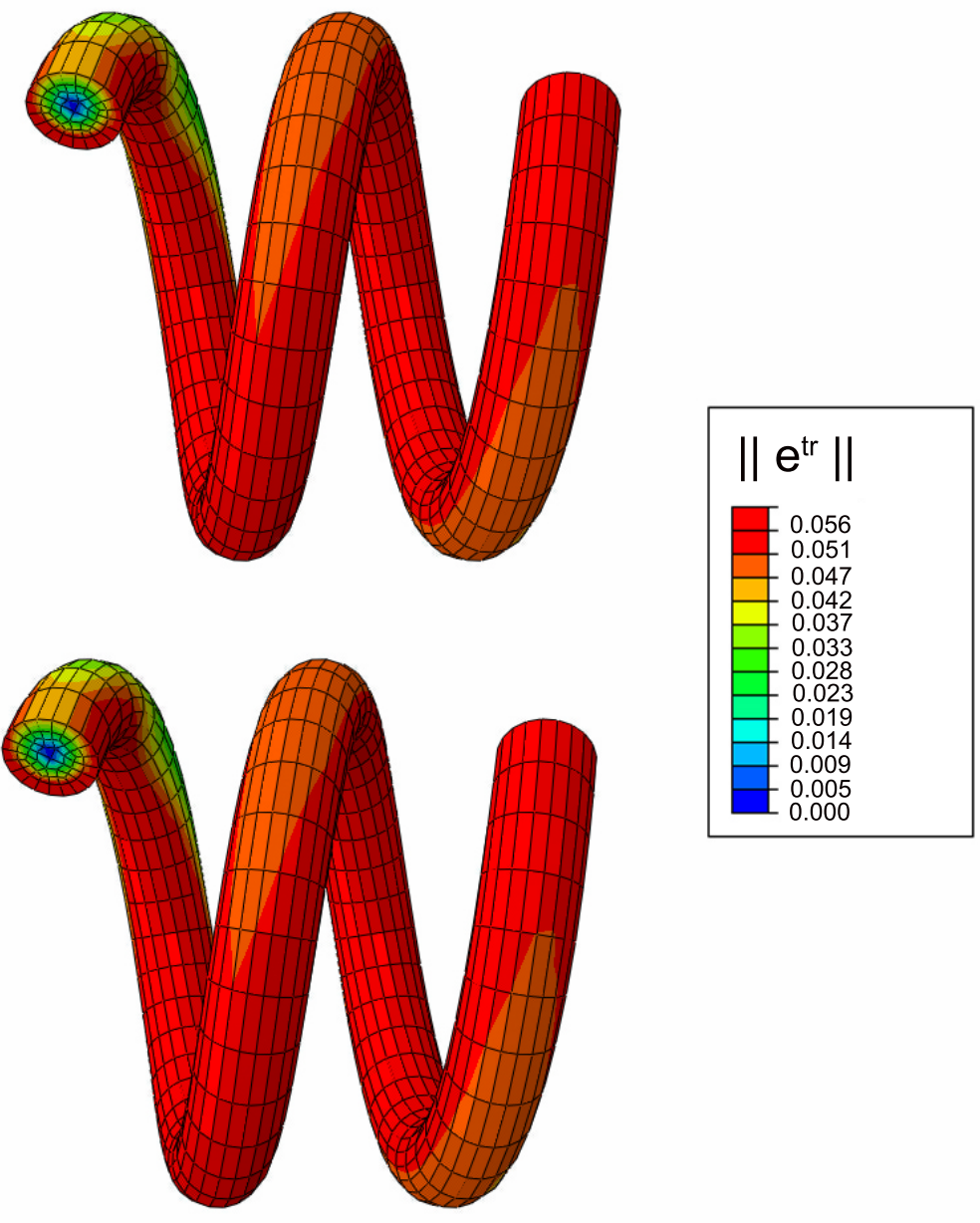

Figure 19: Contour plot of the norm of the transformation strain $\left\|\boldsymbol{e}^{t r}\right\|$ at $169.2 \mathrm{~K}$, obtained with the proposed and classical algorithm for the thermal cycling at constant tensile axial force on the helical spring. 


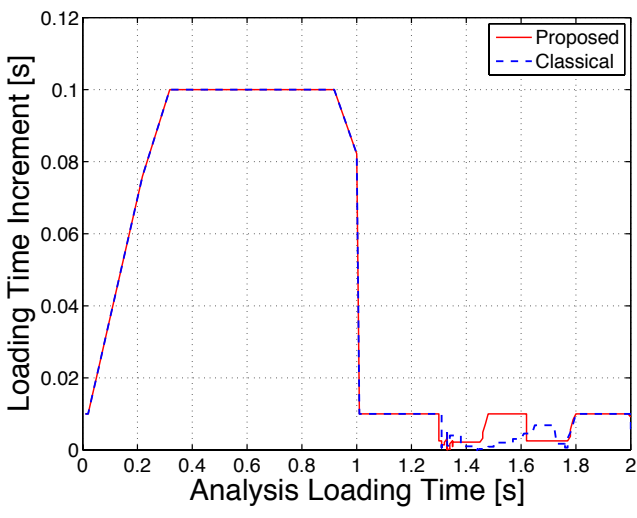

(a)

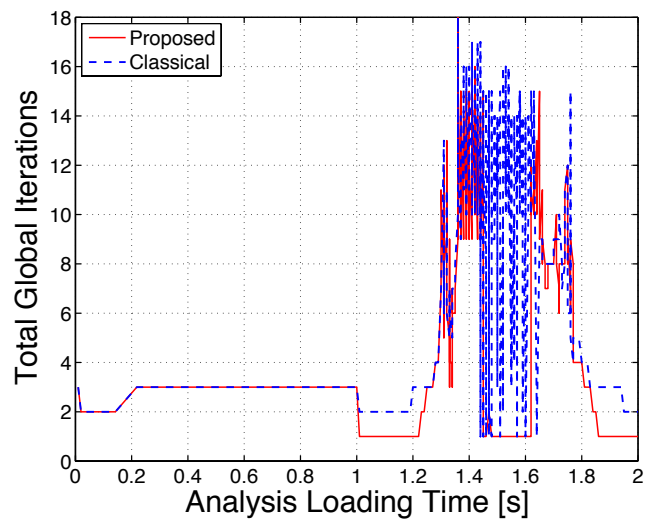

(b)

Figure 20: Thermal cycling at constant tensile axial force on a helical spring. Trends of (a) the loading time increments and (b) the total global iterations during the analysis. Comparison between the proposed and the classical algorithm.

\section{Acknowledgment}

The authors would like to acknowledge Prof. F. Auricchio and Prof. A. Constantinescu for their advice. This work is funded by Fondazione Cariplo, grant number 2013-1779 and by the French National Research Agency (project Fast3D-ANR-11-BS09-012-01).

\section{Appendix A. Variational formulation of the Euler implicit scheme}

Let $\left(\boldsymbol{u}_{n+1}, \boldsymbol{e}_{n+1}^{t r}\right)$ be a solution to the minimization problem (15). We show in the following that $\left(\boldsymbol{u}_{n+1}, \boldsymbol{e}_{n+1}^{t r}\right)$ necessarily satisfies the set of equations (11) that define the Euler implicit scheme. A solution $\left(\boldsymbol{u}_{n+1}, \boldsymbol{e}_{n+1}^{t r}\right)$ to the minimization problem (15) necessarily satisfies the optimality condition

$$
(0,0) \in \partial \mathcal{F}\left(\boldsymbol{u}_{n+1}, \boldsymbol{e}_{n+1}^{t r}\right)
$$

where $\partial \mathcal{F}$ is the subdifferential of $\mathcal{F}$ (Brézis, 1972; Rockafellar, 1970). Observe that $\mathcal{F}$ is differentiable with respect to $\boldsymbol{u}$. Eq. (A.1) can be rewritten as

$$
\begin{aligned}
& 0=\partial \boldsymbol{u} \mathcal{F}\left(\boldsymbol{u}_{n+1}, \boldsymbol{e}_{n+1}^{t r}\right), \\
& 0 \in \partial_{\boldsymbol{e}^{t r}} \mathcal{F}\left(\boldsymbol{u}_{n+1}, \boldsymbol{e}_{n+1}^{t r}\right),
\end{aligned}
$$


where $\partial \boldsymbol{u} \mathcal{F}$ is the derivative of the $\mathcal{F}$ with respect to $\boldsymbol{u}$, and $\partial_{\boldsymbol{e}^{t r}} \mathcal{F}$ is the subdifferential of $\mathcal{F}$ with respect to $\boldsymbol{e}^{t r}$. Reproducing the classical reasoning used in linear elasticity, Eq. (A.2) leads to Eqs. (11.1-2).

Eq. (A.3) needs to be treated more carefully because $\mathcal{F}$ is only subdifferentiable with respect to $\boldsymbol{e}^{t r}$. Since $\boldsymbol{e}^{t r}$ is free from differential constraints, first observe that (A.3) gives the local equation

$$
0 \in \partial_{\boldsymbol{e}^{t r}}(\Psi+\Phi)\left(\boldsymbol{u}_{n+1}(\boldsymbol{x}), \boldsymbol{e}_{n+1}^{t r}(\boldsymbol{x})\right) \forall \boldsymbol{x} \in \Omega
$$

where $\Phi\left(\boldsymbol{e}^{t r}\right)=R_{Y}\left\|\boldsymbol{e}^{t r}-\boldsymbol{e}_{n}^{t r}\right\|$. Further note from (2) that $\Psi$ can be written as $\Psi=\Psi_{0}+\Psi_{1}+\mathcal{I}_{\varepsilon_{L}}$ with $\Psi_{0}=(1 / 2) \kappa \theta^{2}+G\left\|\boldsymbol{e}-\boldsymbol{e}^{t r}\right\|^{2}+(1 / 2) h\left\|\boldsymbol{e}^{t r}\right\|^{2}$ and $\Psi_{1}=\tau_{M}\left\|e^{t r}\right\|$. Since all the functions $\Phi, \Psi_{0}, \Psi_{1}, \mathcal{I}_{\varepsilon_{L}}$ are proper, lower-semicontinuous and convex, we have (Brézis, 1972; Rockafellar, 1970)

$$
\partial_{\boldsymbol{e}^{t r}}(\Psi+\Phi)=\partial_{\boldsymbol{e}^{t r}} \Psi_{0}+\partial_{\boldsymbol{e}^{t r}} \Psi_{1}+\partial_{\boldsymbol{e}^{t r}} \mathcal{I}_{\varepsilon_{L}}+\partial_{\boldsymbol{e}^{t r}} \Phi
$$

The expressions of $\partial_{e^{t r}} \Psi_{1}$ and $\partial_{\boldsymbol{e}^{t r}} \mathcal{I}_{\varepsilon_{L}}$ have been given in Sect. 2.1. We have

$$
\partial_{\boldsymbol{e}^{t r}} \Psi_{0}\left(\boldsymbol{e}_{n+1}^{t r}\right)=-\boldsymbol{s}_{n+1}+h \boldsymbol{e}_{n+1}^{t r}
$$

and

$$
\partial_{\boldsymbol{e}^{t r}} \Phi\left(\boldsymbol{e}_{n+1}^{t r}\right)= \begin{cases}R_{Y} \frac{\boldsymbol{e}_{n+1}^{t r}-\boldsymbol{e}_{n}^{t r}}{\left\|\boldsymbol{e}_{n+1}^{t r}-\boldsymbol{e}_{n}^{t r}\right\|} & \text { if } \boldsymbol{e}_{n+1}^{t r} \neq \boldsymbol{e}_{n}^{t r}, \\ \left\{R_{Y} \tau: \operatorname{tr} \tau=0,\|\tau\| \leq 1\right\} & \text { if } \boldsymbol{e}_{n+1}^{t r}=\boldsymbol{e}_{n}^{t r}\end{cases}
$$

Hence, defining $\boldsymbol{X}_{n+1}$ as in (11.3)-(11.5), Eq. (A.3) gives

$$
\mathbf{0} \in-\boldsymbol{X}_{n+1}+\partial_{\boldsymbol{e}^{t r}} \Phi\left(\boldsymbol{e}_{n+1}^{t r}\right) .
$$

Using (A.5), Eq. (A.6) can be seen to be equivalent to (11.4)(11.6). This completes the proof that the stationarity conditions (A.1) coincide with the local equations (11) of the Euler implicit scheme.

\section{Appendix B. Derivation of the tangent stiffness operator}

From (42) we have

$$
\frac{d \boldsymbol{\sigma}_{n+1}}{d \boldsymbol{\varepsilon}}=\mathbb{C}-2 G \frac{d \boldsymbol{e}_{n+1}^{t r}}{d \varepsilon}
$$


Now $\boldsymbol{e}_{n+1}^{t r}$ only depends on $\boldsymbol{\varepsilon}$ through the deviatoric tensor $\boldsymbol{e}^{\prime}=\left(G / G^{\prime}\right) \boldsymbol{e}=$ $\left(G / G^{\prime}\right) \mathbb{K}: \varepsilon$. Hence

$$
\frac{d \boldsymbol{\sigma}_{n+1}}{d \boldsymbol{\varepsilon}}=\mathbb{C}-2 \frac{G^{2}}{G^{\prime}} \frac{d \boldsymbol{e}_{n+1}^{t r}}{d \boldsymbol{e}^{\prime}} \mathbb{K} .
$$

Both for unsaturated and saturated transformation, $\boldsymbol{e}_{n+1}^{t r}$ satisfies an equation of the form

$$
\mathbf{0}=2 G^{\prime}\left(\boldsymbol{e}_{n+1}^{t r}-\boldsymbol{e}^{\prime}\right)+\tau_{M} \frac{\boldsymbol{e}_{n+1}^{t r}}{x}+R_{Y} \frac{\boldsymbol{e}_{n+1}^{t r}-\boldsymbol{e}_{n}^{t r}}{\left\|\boldsymbol{e}_{n+1}^{t r}-\boldsymbol{e}_{n}^{t r}\right\|},
$$

where $x$ is a function of $\boldsymbol{e}_{n+1}^{t r}$. Differentiating (B.3) gives

$$
\begin{aligned}
2 G^{\prime} d \boldsymbol{e}^{\prime}= & -\frac{\tau_{M}}{x^{2}} \boldsymbol{e}_{n+1}^{t r} d x+\left[\left(2 G^{\prime}+\frac{\tau_{M}}{x}+\frac{R_{Y}}{\left\|\boldsymbol{e}_{n+1}^{t r}-\boldsymbol{e}_{n}^{t r}\right\|}\right) \mathbb{I}\right. \\
& \left.-\frac{R_{Y}}{\left\|\boldsymbol{e}_{n+1}^{t r}-\boldsymbol{e}_{n}^{t r}\right\|^{3}}\left(\boldsymbol{e}_{n+1}^{t r}-\boldsymbol{e}_{n}^{t r}\right) \otimes\left(\boldsymbol{e}_{n+1}^{t r}-\boldsymbol{e}_{n}^{t r}\right)\right]: d \boldsymbol{e}_{n+1}^{t r} .
\end{aligned}
$$

Introducing the unit tensor $\tilde{\boldsymbol{b}}=\boldsymbol{b} /\|\boldsymbol{b}\|$, we have by (33)

$$
\left\|\boldsymbol{e}_{n+1}^{t r}-\boldsymbol{e}_{n}^{t r}\right\|=\frac{\|\boldsymbol{b}\|-R_{Y}}{\tau_{M} / x+2 G^{\prime}}, \frac{\boldsymbol{e}_{n+1}^{t r}-\boldsymbol{e}_{n}^{t r}}{\left\|\boldsymbol{e}_{n+1}^{t r}-\boldsymbol{e}_{n}^{t r}\right\|}=\tilde{\boldsymbol{b}} .
$$

Hence (B.4) simplifies as

$$
2 G^{\prime} d \boldsymbol{e}^{\prime}=-\frac{\tau_{M}}{x^{2}} \boldsymbol{e}_{n+1}^{t r} d x+\frac{\left(2 G^{\prime}+\frac{\tau_{M}}{x}\right)}{\|\boldsymbol{b}\|-R_{Y}}\left[\|\boldsymbol{b}\| \mathbb{I}-R_{Y} \tilde{\boldsymbol{b}} \otimes \tilde{\boldsymbol{b}}\right]: d \boldsymbol{e}_{n+1}^{t r} .
$$

At this point is is necessary to distinguish between unsaturated phase transformation.

Appendix B.1. Unsaturated phase transformation $\left(0<\left\|\boldsymbol{e}_{n+1}^{t r}\right\|<\varepsilon_{L}\right)$

For unsaturated phase transformation, we have $x=\left\|\boldsymbol{e}_{n+1}^{t r}\right\|$, hence $d x=$ $-\tilde{\boldsymbol{e}}_{n+1}^{t r}: d \boldsymbol{e}_{n+1}^{t r}$ where $\tilde{\boldsymbol{e}}_{n+1}^{t r}=\boldsymbol{e}_{n+1}^{t r} /\left\|\boldsymbol{e}_{n+1}^{t r}\right\|$. Moreover, we have $\|\boldsymbol{c}\|=\tau_{M}+$ $2 G^{\prime} x$ by (35). Therefore (B.5) becomes

$$
2 G^{\prime} d \boldsymbol{e}^{\prime}=\frac{\|\boldsymbol{b}\|\|\boldsymbol{c}\|}{x\left(\|\boldsymbol{b}\|-R_{Y}\right)} \mathbb{B}: d \boldsymbol{e}_{n+1}^{t r},
$$

where

$$
\mathbb{B}=\mathbb{I}-\frac{R_{Y}}{\|\boldsymbol{b}\|} \tilde{\boldsymbol{b}} \otimes \tilde{\boldsymbol{b}}-\tau_{M} \frac{\left(\|\boldsymbol{b}\|-R_{Y}\right)}{\|\boldsymbol{b}\|\|\boldsymbol{c}\|} \tilde{\boldsymbol{e}}_{n+1}^{t r} \otimes \tilde{\boldsymbol{e}}_{n+1}^{t r} .
$$


It follows that

$$
\frac{d \boldsymbol{e}_{n+1}^{t r}}{d \boldsymbol{e}^{\prime}}=2 G^{\prime} x \frac{\|\boldsymbol{b}\|-R_{Y}}{\|\boldsymbol{b}\|\|\boldsymbol{c}\|} \mathbb{B}^{-1} .
$$

Because of its special structure, the tensor $\mathbb{B}$ can be inverted in closed form, to give:

$$
\mathbb{B}^{-1}=\mathbb{I}+X_{11} \tilde{\boldsymbol{e}}_{n+1}^{t r} \otimes \tilde{\boldsymbol{e}}_{n+1}^{t r}+X_{22} \tilde{\boldsymbol{b}} \otimes \tilde{\boldsymbol{b}}+X_{12}\left(\tilde{\boldsymbol{e}}_{n+1}^{t r} \otimes \tilde{\boldsymbol{b}}+\tilde{\boldsymbol{b}} \otimes \tilde{\boldsymbol{e}}_{n+1}^{t r}\right),
$$

where the scalars $X_{11}, X_{22}, X_{12}$ are defined by (45). Substituting in (B.7) and (B.2) we arrive at the expression (43) for the tangent stiffness operator.

Appendix B.2. Saturated phase transformation $\left(\left\|\boldsymbol{e}_{n+1}^{t r}\right\|=\varepsilon_{L}\right)$

In the case of saturated transformation, the constraint $\left\|\boldsymbol{e}_{n+1}^{t r}\right\|=\varepsilon_{L}$ implies that $\boldsymbol{e}_{n+1}^{t r}: d \boldsymbol{e}_{n+1}^{t r}=0$. Moreover (40) gives $2 G^{\prime}+\tau_{M} / x=\|\boldsymbol{c}\| / \varepsilon_{L}$. Hence projecting (B.5) on $\boldsymbol{e}_{n+1}^{t r}$, we obtain:

$$
2 G^{\prime} d \boldsymbol{e}^{\prime}: \boldsymbol{e}_{n+1}^{t r}=-\frac{\tau_{M}}{x^{2}} \epsilon_{L}^{2} d x-\frac{R_{Y}\|\boldsymbol{c}\|}{\left(\|\boldsymbol{b}\|-R_{Y}\right) \varepsilon_{L}}\left(\tilde{\boldsymbol{b}}: \boldsymbol{e}_{n+1}^{t r}\right)\left(\tilde{\boldsymbol{b}}: d \boldsymbol{e}_{n+1}^{t r}\right)
$$

which gives the expression of $d x$. Substituting the result in (B.5), we obtain the relation

$$
2 G^{\prime}\left(\mathbb{I}-\tilde{\boldsymbol{e}}_{n+1}^{t r} \otimes \tilde{\boldsymbol{e}}_{n+1}^{t r}\right): d \boldsymbol{e}^{\prime}=\frac{\|\boldsymbol{b}\|\|\boldsymbol{c}\|}{\left(\|\boldsymbol{b}\|-R_{Y}\right) \varepsilon_{L}}\left(\mathbb{I}-\frac{R_{Y}}{\|\boldsymbol{b}\|} \boldsymbol{b}_{\perp} \otimes \tilde{\boldsymbol{b}}\right): d \boldsymbol{e}_{n+1}^{t r}
$$

where $\boldsymbol{b}_{\perp}=\tilde{\boldsymbol{b}}-\alpha \tilde{\boldsymbol{e}}_{n+1}^{t r}$ is the projection of $\tilde{\boldsymbol{b}}$ on the orthogonal of $\boldsymbol{e}_{n+1}^{t r}$. Noting that $\boldsymbol{b}: d \boldsymbol{e}_{n+1}^{t r}=\boldsymbol{b}_{\perp}: d \boldsymbol{e}_{n+1}^{t r}$, (B.8) can be equivalently rewritten as

$$
2 G^{\prime}\left(\mathbb{I}-\tilde{\boldsymbol{e}}_{n+1}^{t r} \otimes \tilde{\boldsymbol{e}}_{n+1}^{t r}\right): d \boldsymbol{e}^{\prime}=\frac{\|\boldsymbol{b}\|\|\boldsymbol{c}\|}{\left(\|\boldsymbol{b}\|-R_{Y}\right) \varepsilon_{L}} \mathbb{A}: d \boldsymbol{e}_{n+1}^{t r},
$$

where $\mathbb{A}$ is the symmetric fourth-order tensor defined as:

$$
\mathbb{A}=\mathbb{I}-\frac{R_{Y}}{\|\boldsymbol{b}\|} \boldsymbol{b}_{\perp} \otimes \boldsymbol{b}_{\perp}
$$

Hence

$$
\frac{d \boldsymbol{e}_{n+1}^{t r}}{d \boldsymbol{e}^{\prime}}=2 G^{\prime} \frac{\left(\|\boldsymbol{b}\|-R_{Y}\right) \varepsilon_{L}}{\|\boldsymbol{b}\|\|\boldsymbol{c}\|} \mathbb{A}^{-1}:\left(\mathbb{I}-\tilde{\boldsymbol{e}}_{n+1}^{t r} \otimes \tilde{\boldsymbol{e}}_{n+1}^{t r}\right) .
$$

The tensor $\mathbb{A}$ can be inverted in closed form to give:

$$
\mathbb{A}^{-1}=\mathbb{I}+Y \boldsymbol{b}_{\perp} \otimes \boldsymbol{b}_{\perp}
$$


with

$$
Y=\frac{R_{Y}}{\|\boldsymbol{b}\|-R_{Y}\left(1-\alpha^{2}\right)} .
$$

Replacing in (B.9) and recalling that $\boldsymbol{b}_{\perp}: \tilde{\boldsymbol{e}}_{n+1}^{t r}=0$, we find:

$$
\frac{d \boldsymbol{e}_{n+1}^{t r}}{d \boldsymbol{e}^{\prime}}=2 G^{\prime} \frac{\left(\|\boldsymbol{b}\|-R_{Y}\right) \varepsilon_{L}}{\|\boldsymbol{b}\|\|\boldsymbol{c}\|}\left(\mathbb{I}-\tilde{\boldsymbol{e}}_{n+1}^{t r} \otimes \tilde{\boldsymbol{e}}_{n+1}^{t r}+Y \boldsymbol{b}_{\perp} \otimes \boldsymbol{b}_{\perp}\right) .
$$

Substituting in (B.2) gives the expression (44) for the tangent stiffness operator.

\section{Bibliography}

Abaqus, 2010. Abaqus, Analysis Users Manual, 2010, Dassault Systemes of America Corp, Woodland Hills, CA.

Arghavani, J., Auricchio, F., Naghdabadi, R., Reali, A., 2011a. An improved, fully symmetric, finite-strain phenomenological constitutive model for shape memory alloys. Finite Elements in Analysis and Design 47, 166174 .

Arghavani, J., Auricchio, F., Naghdabadi, R., Reali, A., 2011b. On the robustness and efficiency of integration algorithms for a $3 \mathrm{~d}$ finite strain phenomenological sma constitutive model. International Journal for Numerical Methods in Engineering 85 (1), 107-134.

Arghavani, J., Auricchio, F., Naghdabadi, R., Reali, A., Sohrabpour, S., 2010. A 3-d phenomenological constitutive model for shape memory alloys under multiaxial loadings. International Journal of Plasticity 26, 976-991.

Artioli, E., Bisegna, P., 2015. An incremental energy minimization state update algorithm for $3 \mathrm{~d}$ phenomenological internal-variable sma constitutive models based on isotropic flow potentials. International Journal of Numerical Methods in Engineering 105 (3), 197-220.

Auricchio, F., Boatti, E., Reali, A., Stefanelli, U., 2016. Gradient structures for the thermomechanics of shape-memory materials. Computer Methods in Applied Mechanics and Engineering 299, 440 - 469.

Auricchio, F., Bonetti, E., 2013. A new flexible 3d macroscopic model for shape memory alloys. Discrete Contin. Dyn. Syst. Ser. S 6 (2), 277-291. 
Auricchio, F., Bonetti, E., Scalet, G., Ubertini, F., 2014a. Theoretical and numerical modeling of shape memory alloys accounting for multiple phase transformations and martensite reorientation. International Journal of Plasticity 59, 30-54.

Auricchio, F., Conti, M., Ferraro, M., Morganti, S., Reali, A., Taylor, R., 2015. Innovative and efficient stent flexibility simulations based on isogeometric analysis. Computer Methods in Applied Mechanics and Engineering 295, 347-361.

Auricchio, F., Morganti, S., Reali, A., 2009a. SMA numerical modeling versus experimental results. In: Proceedings of European Symposium on Martensitic Transformations (ESOMAT 2009). pp. 1-6.

Auricchio, F., Petrini, L., 2004. A three-dimensional model describing stresstemperature induced solid phase transformations: solution algorithm and boundary value problems. International Journal for Numerical Methods in Engineering 61, 807-836.

Auricchio, F., Reali, A., Stefanelli, U., 2007. A three-dimensional model describing stress-induces solid phase transformation with residual plasticity. Int. J. Plasticity 23, 207-226.

Auricchio, F., Reali, A., Stefanelli, U., 2009b. A macroscopic 1d model for shape memory alloys including asymmetric behaviors and transformationdependent elastic properties. Comput. Methods Appl. Mech. Eng. 198, 1631-1637.

Auricchio, F., Scalet, G., Urbano, M., 2014b. A numerical/experimental study of nitinol actuator springs. Journal of Materials Engineering and Performance 23 (7), 2420-2428.

Barrera, N., Biscari, P., Urbano, M., 2014. Macroscopic modeling of functional fatigue in shape memory alloys. Eur. J. Mech. A/Solids 45, 101-109.

Bartel, T., Hackl, K., 2009. A micromechanical model for martensitic transformations in shape-memory alloys based on energy relaxation. ZAMM 89, 792809 . 
Bartel, T., Hackl, K., 2010. Multiscale modeling of martensitic phase transformations: on the numerical determination of heterogeneous mesostructures within shape-memory alloys induced by precipitates. Techn. Mech. 30,324342 .

Bartel, T., Menzel, A., Svendsen, B., 2011. Thermodynamic and relaxationbased modeling of the interaction between martensitic phase transformations and plasticity. Journal of the Mechanics and Physics of Solids 59, 1004-1019.

Boatti, E., Ferraro, M., Scalet, G., Auricchio, F., 2016. Development of an effective and user-friendly numerical framework for the simulation of complex smart material components and devices. In: Abstract of the First International Conference on Materials Design and Applications (MDA 2016). Porto, Portugal.

Brézis, H., 1972. Opérateurs maximum monotones et semigroupes de contractions dans les espaces de Hilbert. North-Holland, Amsterdam.

Carstensen, C., Hackl, K., Mielke, A., 2002. Non-convex potentials and microstructures in finite-strain plasticity. Proceedings of the Royal Society A: Mathematical, Physical and Engineering Science 458, 299-317.

Chemisky, Y., Chatzigeorgiou, G., Kumar, P., Lagoudas, D., 2014. A constitutive model for cyclic actuation of high-temperature shape memory alloys. Mechanics of Materials 68, 120-136.

Cisse, C., Zaki, W., Zineb, T. B., 2016. A review of constitutive models and modeling techniques for shape memory alloys. International Journal of Plasticity 76, $244-284$.

Coleman, B. D., Noll, W., 1963. The termodynamics of elastic mmaterial with heat conduction and viscosity. Arch. Ration. Mech. Anal. (13), 167178.

Dhote, R., Gomez, H., Melnik, R., Zu, J., 2015. 3d coupled thermomechanical phase-field modeling of shape memory alloy dynamics via isogeometric analysis. Computers \& Structures 154, 48 - 58. 
Evangelista, V., Marfia, S., Sacco, E., 2009. Phenomenological 3d and 1d consistent models for shape-memory alloy materials. Comput. Mech. 44, 405-421.

Grandi, D., Stefanelli, U., 2014. A phenomenological model for microstructure-dependent inelasticity in shape-memory alloys. Meccanica 49, 2265-2283.

Grandi, D., Stefanelli, U., 2015. The souza-auricchio model for shape-memory alloys. Discrete and Continuous Dynamical Systems - Series S 8 (4), 723747.

Gurtin, M., 1981. An Introduction to Continuum Mechanics. Academic Press, New York.

Hartl, D., Chemisky, Y., Meraghni, F., 2014. Three-dimensional constitutive model considering transformation-induced damage and resulting fatigue failure in shape memory alloys. In: Proc SPIE 9058, behavior and mechanics of multifunctional materials and composites. p. 905805.

Hartl, D., Lagoudas, D., 2009. Constitutive modeling and structural analysis considering simultaneous phase transformation and plastic yield in shape memory alloys. Smart Materials and Structures 18, 117.

Jähne, R., 2012. Multiaxial mechanical characterization and constitutive modeling of superelastic sheets for solid-state hinges. Ph.D. thesis, ETH Zurich.

Jani, J., Leary, M., Subic, A., Gibson, M., 2014. A review of shape memory alloy research, applications and opportunities. Materials \& Design 56, 1078-1113.

Kiefer, B., Bartel, T., Menzel, A., 2012. Implementation of numerical integration schemes for the simulation of magnetic sma constitutive response. Smart Materials and Structures 21, 1-8.

Korelc, J., 2002. Multi-language and multi-environment generation of nonlinear finite element codes. Engineering with Computers 18 (4), 312-327.

Lagoudas, D., 2008. Shape Memory Alloys: Modeling and Engineering Applications. Springer. 
Lagoudas, D. C., Hartl, D. J., Chemisky, Y., Machado, L., Popov, P., 2012. Constitutive model for the numerical analysis of phase transformation in polycrystalline shape memory alloys. International Journal of Plasticity 32-33, 155-183.

Lexcellent, C., Leclercq, S., Gabry, B., Bourbon, G., 2000. The two way shape memory effect of shape memory alloys: an experimental study and a phenomenological model. International Journal of Plasticity 16, 11551168.

Meisel, N., Elliott, A., Williams, C., 2015. A procedure for creating actuated joints via embedding shape memory alloys in polyjet $3 \mathrm{~d}$ printing. Journal of Intelligent Material Systems and Structures 26 (12), 1498-1512.

Miehe, C., Schotte, J., Lambrecht, M., 2002. Homogenization of inelastic materials at finite strains based on incremental variational principles. application to the texture analysis of polycrystals. Journal of the Mechanics and Physics of Solids 50, 2123-2167.

Ortiz, M., Repetto, E., 1999. Nonconvex energy minimization and dislocation structures in ductile single crystals. Journal of the Mechanics and Physics of Solids 47 (2), 397-462.

Pan, H., Thamburaja, P., Chau, F., 2007. Multi-axial behavior of shapememory alloys undergoing martensitic reorientation and detwinning. International Journal of Plasticity 23 (4), 711-732.

Peigney, M., Seguin, J., 2013. An incremental variational approach to coupled thermo-mechanical problems in anelastic solids. Application to shapememory alloys. International Journal of Solids and Structures 50 (24), 4043-4054.

Peigney, M., Seguin, J., Hervé-Luanco, E., 2011. Numerical simulation of shape memory alloys structures using interior-point methods. Int. J. Solids Struct. 48, 2791-2799.

Peraza-Hernandez, E.and Hartl, D., Galvan, E.and Malak, R., 2013. Design and optimization of a shape memory alloy-based self-folding sheet. Journal of Mechanical Design, Transactions of the ASME 135 (11), 1-11. 
Popov, P., Lagoudas, D., 2007. A 3d constitutive model for shape memory alloys incorporating pseudoelasticity and detwinning of self-accommodated martensite. International Journal of Plasticity 23, 1679-1720.

Qidwai, M. A., Lagoudas, D., 2000. Numerical implementation of a shape memory alloy thermomechanical constitutive model using return mapping algorithms. International Journal for Numerical Methods in Engineering 47, 1123-1168.

Quarteroni, A., 2009. Numerical Models for Differential Problems. Springer.

Rockafellar, R. T., 1970. Convex Analysis. Princeton University Press.

Scalet, G., Auricchio, F., Hartl, D., 2015. Efficiency and effectiveness of implicit and explicit approaches for the analysis of shape-memory alloy bodies. Journal of Intelligent Material Systems and Structures x (x), x.

Sedlák, P., Frost, M., Benesová, B., Zineb, T. B., Sittner, P., 2012. Thermomechanical model for niti-based shape memory alloys including r-phase and material anisotropy under multi-axial loadings. International Journal of Plasticity 39, 132-151.

Simo, J., Hugues, T., 1998. Computational Inelasticity. Springer.

Souza, A., Mamiya, E., Zouain, N., 1998. Three-dimensional model for solids undergoing stress-induced phase transformations. European Journal of Mechanics A/Solids 17, 789-806.

Stebner, A., Brinson, L., 2013. Explicit finite element implementation of an improved three dimensional constitutive model for shape memory alloys. Computer Methods in Applied Mechanics and Engineering 257, 17-35.

Stupkiewicz, S., Petryk, H., 2013. A robust model of pseudoelasticity in shape memory alloys. International Journal of Numerical Methods in Engineering 93, 747-769.

Tanaka, K., Nagaki, S., 1982. A thermomechanical description of materials with internal variables in the process of phase transitions. Ing. Archiv. 51 (5), 287-299. 
Wayman, C. M., 1989. Engineering Aspects of Shape Memory Alloys. Butterworth-Heinemann (Boston, MA)1, Ch. An introduction to martensite and shape memory, pp. 3-20.

Wilkins, M., 1964. Calculation of elastic-plastic flow. In: Methods of Computational Physics 3. Academic Press New York.

Zaki, W., 2012. Time integration of a model for martensite detwinning and reorientation under non-proportional loading using Lagrange multipliers. International Journal of Solids and Structures 49 (21), 2951-2961.

Zaki, W., Moumni, Z., 2007. A three-dimensional model of the thermomechanical behavior of shape memory alloys. Journal of the Mechanics and Physics of Solids 55, 2455-2490. 